\title{
Structural and vibrational spectral studies on hydrogen bonded salts: 4-chloroanilinium maleate and nitrate
}

\author{
R ANITHA $^{\mathrm{a}}$, M GUNASEKARAN ${ }^{\mathrm{a}}$, S SURESH KUMAR ${ }^{\mathrm{b}}$ and S ATHIMOOLAM ${ }^{\mathrm{b}, *}$ \\ ${ }^{a}$ Department of Physics, Regional Centre, Anna University Tirunelveli Region, Tirunelveli 627 007, India \\ ${ }^{b}$ Department of Physics, University College of Engineering, Nagercoil, Anna University, \\ Nagercoil 629 004, India \\ e-mail: athi81s@yahoo.co.in
}

MS received 25 October 2014; revised 17 May 2015; accepted 19 May 2015

\begin{abstract}
In the present study, proton transfer from nitric and maleic acids to amine group (4-chloroaniline) led to hydrogen bonded crystals of 4-chloroanilinium maleate (4CAM) and 4-chloroanilinium nitrate (4CAN) which are investigated by the experimental and theoretical approaches. The molecular structures of these two compounds were optimized with the Density Functional Theory (DFT) using B3LYP function and the HartreeFock (HF) level with a6-311++G(d,p) basis set. Geometrical parameters of the molecules were also analyzed along with their intermolecular hydrogen bond, which tailors the ions. These analyses show that present molecules are stabilized through the $\mathrm{N}-\mathrm{H} \cdots \mathrm{O}$ and $\mathrm{O}-\mathrm{H} \cdots \mathrm{O}$ hydrogen bonds. The vibrational modes were computed by quantum chemical methods. Further, these modes are investigated by FT-IR and FT-Raman spectroscopy in the range of 4000-400 $\mathrm{cm}^{-1}$. The optimized molecular geometry and computed vibrational spectra are compared with experimental results, which show significant agreement. The natural bond orbital (NBO) analysis was carried out to interpret hyperconjucative interaction and intramolecular charge transfer (ICT). This analysis gives the precise insight into the nature of H-bond interactions. The chemical hardness, electronegativity and chemical potential of the molecules were determined by HOMO-LUMO plot. The frontier molecular orbitals have small band gap value, which signify the possible biological/pharmaceutical activity of the compounds.
\end{abstract}

Keywords. FT-IR spectrum; FT-Raman spectrum; Vibrational analysis; quantum chemical analysis; HOMOLUMO; NBO.

\section{Introduction}

One of the most important reactions in chemistry and biochemistry is proton transfer between donor and acceptor. ${ }^{1}$ These interactions are playing an important role in various chemical and biological processes such as stabilizing the biomolecular structures, ${ }^{2}$ controlling the speed of the enzymatic reactions ${ }^{3}$ and constructing supramolecular structures. ${ }^{4}$ In most of the cases, the applications are ensued due to the intra- and intermolecular interactions particularly with the hydrogen bonds, which have more importance in the areas of molecular recognition, crystal engineering research and supramolecular chemistry. Understanding of these non-covalent interactions has proved to be the most valuable because of its strength and directional properties. ${ }^{5}$ The strength and directionality of hydrogen bonding interactions are responsible for solid-state formation and other physical properties of the system. ${ }^{6}$ Also, we continuously seek to

*For correspondence identify hydrogen bond-enriched assemblies by means of efficient organic hydrogen bonding synthons.

Substituted anilines are known to be candidates for supramolecular synthons as they have both donor and acceptor sites, viz., nitrogen and chlorine atoms in the present case. The present work was attempted with nitrate and maleate salts of 4-chloroanilinium crystal. Though the single-crystal XRD clearly predicts the molecular structure and packing tendency of the molecule in the solid crystalline state, the spectral measurement gives an account of the strength of the intermolecular interactions by means of shifting of wavenumbers and the intensity of the peaks. Further, the molecular geometry obtained from the crystallographic data is a wellsuited input for the quantum chemical calculations. By which it is possible to optimize the molecule in different environments and the corresponding vibrational modes can be calculated. The hyperconjugative interactions and band gap values can be inferred from natural bond orbitals (NBOs) and frontier molecular orbital (FMO) studies. Hence, a combined crystallographic, spectroscopic and quantum chemical calculations were 
attempted on 4-chloroanilinium maleate (4CAM) and 4-chloroanilinium nitrate (4CAN), and the results are summarized.

\section{Experimental}

\subsection{Crystal Growth}

Crystals of 4CAM and 4CAN were synthesized from aqueous mixtures containing 4-chloroaniline with maleic and nitric acid in the 1:1 stoichiometric ratio at room temperature by slow evaporation method, respectively. After a week, light yellow colour crystals of 4CAM and 4CAN were obtained. The qualities of the crystals were improved by recrystallization.

\subsection{Density Measurement}

The density of the grown crystals were measured by sink and swim method (flotation technique) using a liquid mixture of carbon tetrachloride and xylene. Initially, carbon tetrachloride $(5 \mathrm{~mL})$ was taken in a test tube and a good-quality three-dimensional crystal was placed on it. Due to high density of the liquid, the crystal began to float. Then, drops of xylene were added drop by drop with continuous agitation to get uniform density over the liquid. When the density of the crystal matches that of liquid mixture, the crystal levitates to the middle of the test tube. Then, the density of the liquid was found with specific gravity bottle from the concept of relative density. Thus, the densities of the crystals are found to be $1.495 \mathrm{mg} . \mathrm{m}^{-3}$ for $4 \mathrm{CAM}$ and $1.465 \mathrm{mg} \cdot \mathrm{m}^{-3}$ for $4 \mathrm{CAN}$.

\subsection{Single-Crystal XRD Studies}

The entire crystallographic calculations of 4CAM were performed done using single-crystal X-ray diffraction by Bruker SMART APEX CCD area detector diffractometer (graphite- monochromated, $\mathrm{MoK}_{\alpha}=0.71073 \AA$ ). A good-quality single-crystal was selected from the grown crystals and used for the single crystal X-ray diffraction studies. Consequently, the full data collection of the crystal was carried out. The cell refinement and data reduction were done with XCAD4 software. ${ }^{7}$ The structure was solved and refined with the SHELXL97 program. ${ }^{8}$ All the $\mathrm{H}$ atoms were discernible in the difference electron density maps. Nevertheless, the aryl $\mathrm{H}$ atoms were constrained and refined in the riding atom approximation: $\mathrm{C}-\mathrm{H}=0.93, \AA$ and $\mathrm{U}_{\text {iso }}(\mathrm{H})=1.2 \mathrm{U}_{\mathrm{eq}}(\mathrm{C})$. The other $\mathrm{H}$ atoms, which are involved in the $\mathrm{N}-\mathrm{H} \cdots \mathrm{O}$ and $\mathrm{O}-\mathrm{H}$. . . O hydrogen bonds, were located from electronic density map and refined isotropically. Graphical molecular illustrations were done with ORTEP3 for Windows ${ }^{9}$ and Mercury ${ }^{10}$ packages.

Though good-quality crystals of 4CAN were obtained, the attempts of single crystal X-ray diffraction was not fruitful due to the hygroscopic nature of the crystal.

\subsection{Vibrational Spectroscopic Measurements}

A Jasco spectrometer FT-IR, model 410 under a resolution of $4 \mathrm{~cm}^{-1}$ and with a scanning speed of $2 \mathrm{~mm} / \mathrm{sec}$ was used for IR spectral measurements. The samples were prepared using pellet technique and the spectrum was recorded over the range $4000-400 \mathrm{~cm}^{-1}$. The FTRaman spectrum was recorded in the frequency range of 4000-50 $\mathrm{cm}^{-1}$ using a BRUKER RFS 27 FT-Raman Microscope module with the resolution of $2 \mathrm{~cm}^{-1}$. The $\mathrm{Nd}$ : YAG Laser source was operated at $1064 \mathrm{~nm}$ for the excitation.

\subsection{Computational details}

The geometrical optimization for 4CAM and 4CAN molecules was carried out theoretically by $6-311++$ $\mathrm{G}(\mathrm{d}, \mathrm{p})$ level on an Intel Core i5/3.20 GHz computer using Gaussian $09 \mathrm{~W}^{11}$ program package without any constraint. ${ }^{12}$ Initial geometry was taken from the singlecrystal X-ray studies and it was minimized (optimized) by Hartree-Fock (HF) method using the 6-311++G (d,p) basis set. The molecular geometries were also optimized by the Density Functional Theory (DFT) using the Becke's three-parameters exchange functional (B3) $)^{13}$ in combination with the Lee-Yang-Parr correlation functional (LYP). ${ }^{14}$ It is accepted as a cost-effective approach for the computation of molecular structure, vibrational frequencies and energies of optimized structures. The optimized structural parameters were used in the vibrational frequency calculations at the same level to characterize all stationary points as minima. Then vibrationally averaged nuclear positions of the structure were used for harmonic vibrational frequency calculations resulting in IR and Raman frequencies. Finally, the calculated normal mode vibrational frequencies provide thermodynamic properties through the principle of statistical mechanics. By combining the analysis preformed using the GAUSSVIEW program ${ }^{15}$ with symmetry considerations, vibrational frequency assignments were made with a high degree of accuracy. There is always some ambiguity in defining internal coordination. However, the defined coordinate from the complete set matches quite well with the motions observed using the GAUSSVIEW program. Also, the NBO calculations were carried out using HF and DFT methods 
with the $6-311++\mathrm{G}(\mathrm{d}, \mathrm{p})$ basis set to get more detailed information about the chemical bonds and hydrogen bonding interaction within the ions. The FMOs have been computed and analyzed by HF and DFT method with the $6-311++\mathrm{G}(\mathrm{d}, \mathrm{p})$ basis set.

\section{Results and Discussions}

\subsection{Molecular Geometry}

The solid-state molecular and crystal structures of 4CAM are illustrated from the single-crystal XRD studies. The asymmetric part of the 4CAM contains a 4-chloroanilinium cation and a maleate anion shown in figure 1 . As 4CAN is prepared with 4-chloroaniline and nitric acid, the crystals are expected to possess 4-chloroanilinium cation and a nitrate anion in the asymmetric part. The optimized structures of both the molecular assemblies are shown in figure 2 . In both the compounds, the transfer of proton from the inorganic and organic acid leads to protonation on the $\mathrm{NH}_{2}$ sites of the 4-chloroaniline and it forms the 4-chloroanilinium cation. This protonation on the $\mathrm{N}$ site of the cation is confirmed from the elongated $\mathrm{C}-\mathrm{N}$ bond distance [1.456 (3) $\AA$ ] in 4CAM. ${ }^{16}$ The protonated bond distance is compared with the theoretically computed bond distances. The optimized C-N bond distances are 1.418 and $1.426 \AA$ in HF and DFT levels, respectively, for 4CAN and 1.415 and $1.425 \AA$ by the HF and DFT levels, respectively, for 4CAM (table 1). In theoretical calculations the $\mathrm{C}-\mathrm{N}$ bond distances of the molecules are observed to be smaller than the single crystal XRD study which is due to the effect of $\mathrm{N}-\mathrm{H}$... O intermolecular hydrogen bonds. Even though the hydrogen atom seems to have deviated from the $\mathrm{N}$ atom of the cation, there must be some attraction between cation and anion which is confirmed by the Mulliken charge distribution analysis. The bond geometry between $\mathrm{N}, \mathrm{H}$ and $\mathrm{O}$ atoms which connect the ion pairs, are shown scheme 1. From the Mulliken charge distribution analysis of the two complexes, $\mathrm{N}$ atom is more electronegative than the $\mathrm{O}$ atom. These $\mathrm{N}$ atoms of the cations are attracting the proton from acids and it forms the $\mathrm{N}-\mathrm{H} \cdots \mathrm{O}$ intermolecular hydrogen bond. The $\mathrm{N}-\mathrm{H} \cdots \mathrm{O}$ angle is $180^{\circ}$ and $179^{\circ}$ at the $\mathrm{HF}$ and DFT/B3LYP methods, respectively, for 4CAN and $172^{\circ}$ in both methods for 4CAM, whereas the experimental counterpart is only $158(2)^{\circ}$. Hence, this intermolecular hydrogen bonding effect is perceived to be dominant in the DFT/B3LYP and HF methods than in the experimental methods.

In 4CAM, the planes of the cation and maleate anion are inclined at an angle $15.6(1)^{\circ}$ in the single crystal

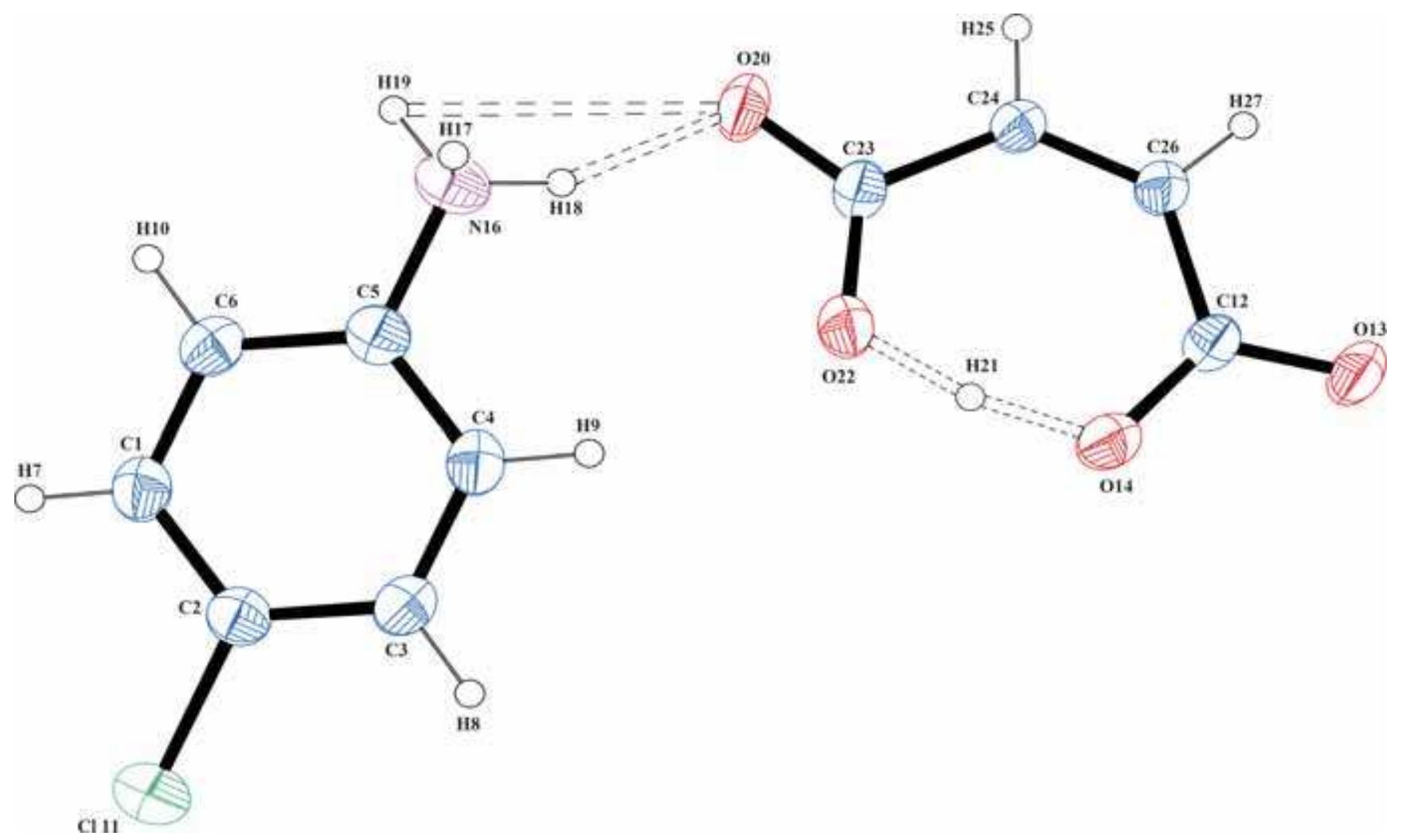

Figure 1. The molecular structure of the 4CAM with the numbering scheme for the atoms and 50\% probability displacement ellipsoids. 

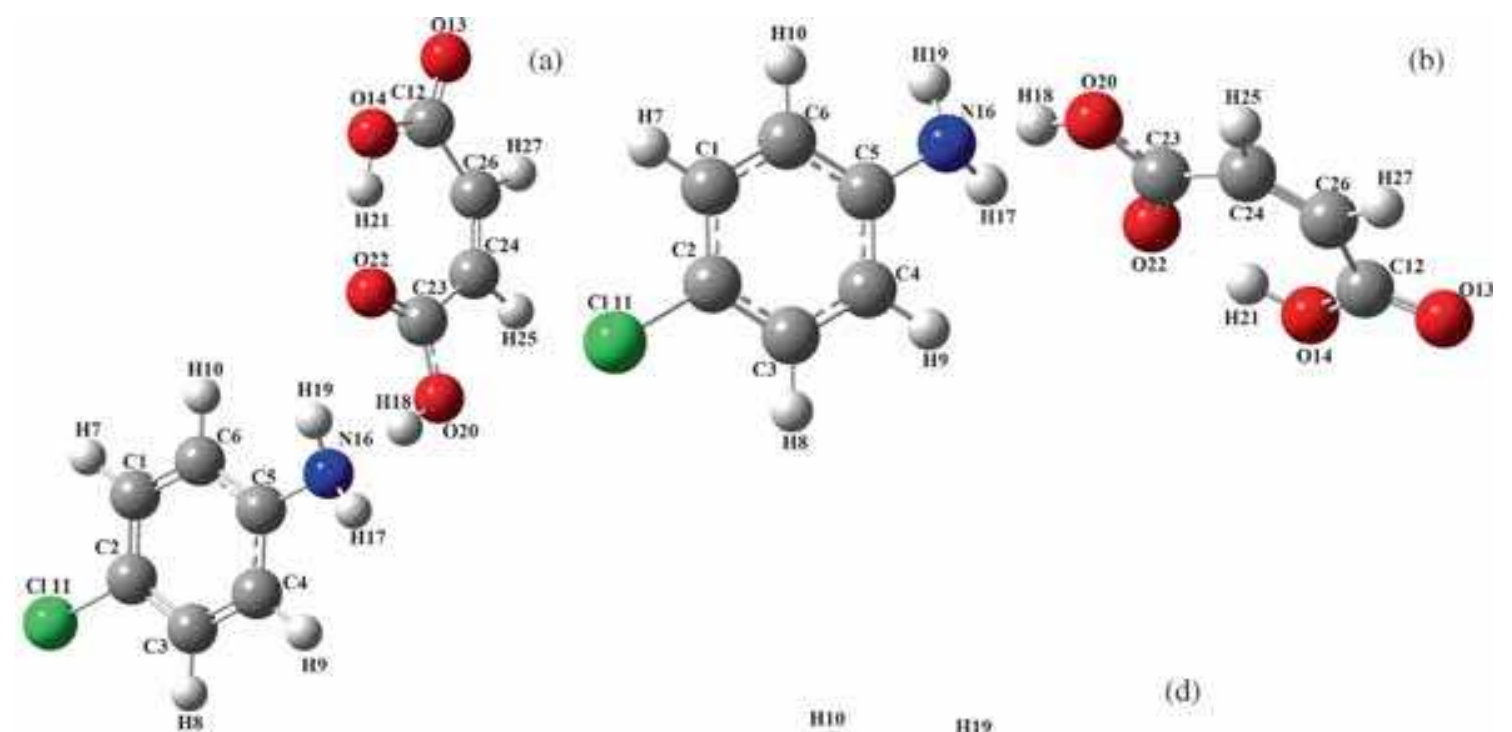

(c)
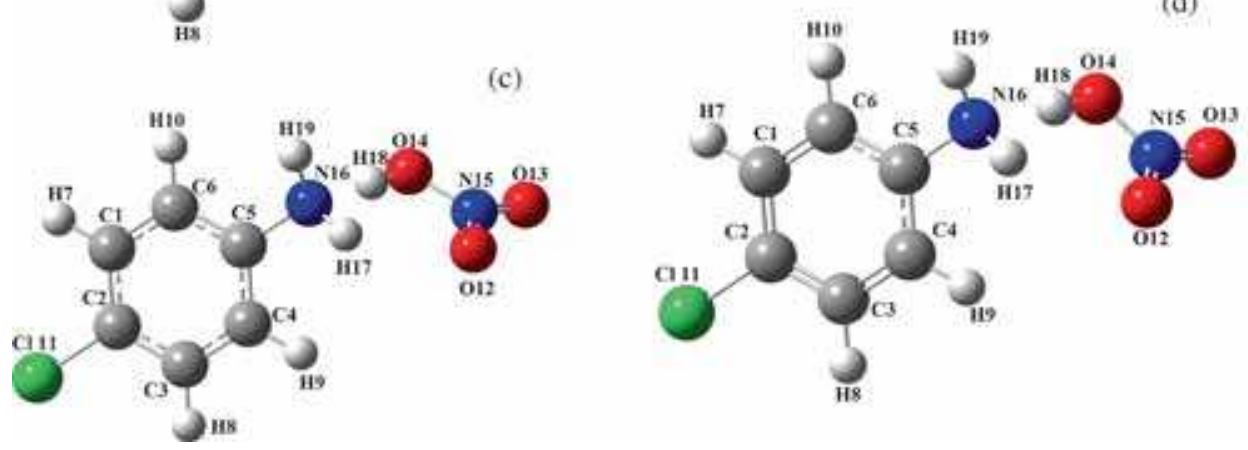

Figure 2. Optimized molecular geometry and atomic numbering scheme by HF (a) DFT; (b) methods for 4CAM and HF; (c) DFT; (d) for 4CAN.

$\mathrm{XRD}$, these planar angles are calculated at $73.6^{\circ}$ and $73.9^{\circ}$ in HF and DFT methods respectively. These deviations occur due to the reorientation of $\mathrm{N}-\mathrm{H}$. . O intermolecular hydrogen bond. The $\mathrm{C}-\mathrm{O}$ bond distances of the carboxylate group from the maleate anion are 1.182$1.302 \AA$ at the HF level and $1.209-1.330 \AA$ by the B3LYP method, whereas in experimental methods it is 1.234 (2)-1.267 (2) $\AA$ (table 2). The planarity of the maleate anion is confirmed by the torsion angles.

In 4CAN, the nitrate group exists in a planar orientation with the $\mathrm{O}-\mathrm{N}-\mathrm{O}$ angles varying from 115.5 to $127.6^{\circ}$ in HF level and from 115.1 to $128^{\circ}$ in the DFT/ B3LYP method. The average bond lengths varied at $1.189-1.318$ and $1.222-1.379 \AA$ in the HF and DFT/ B3LYP methods respectively (table 3 ). One of the N-O bond distances (1.318 $\AA$ in HF and $1.379 \AA$ in B3LYP) in the nitrate anion increases more than the literature value $(\sim 1.240 \AA)$. This elongation is due to the $\mathrm{N}-$ $\mathrm{H}$... O hydrogen bond between the cation and anion. It shows the possible charge transfer between donor $\mathrm{N}$ and acceptor $\mathrm{O}$ atoms. This hydrogen bonding effect is less in the HF level, which is revealed in the computed spectra. The $-\mathrm{NH}_{2}$ stretching mode of $\mathrm{NH}_{3}^{+}$group is observed to be low-intense peak in the HF level, whereas it is the strongest among the computed vibrational peaks in the DFT/B3LYP level.

The optimized $\mathrm{C}-\mathrm{C}$ bond lengths of phenyl ring are in the falling range $1.381-1.392 \AA$, according in the theoretical calculations, whereas the single-crystal $\mathrm{XRD}$ reveals the $\mathrm{C}-\mathrm{C}$ bond lengths in the range of 1.368-1.376 A. On comparison, it is observed that most of the optimized bond lengths are slightly deviated from the experimental values. Because theoretical calculations were carried out for the molecules in the free gaseous phase and the experimental results corresponding to the molecules are in the solid crystalline state.

\subsection{Mulliken Charge Analysis}

In general, Mulliken atomic charge calculation has an important application of quantum chemical calculations to molecular systems. It plays a vital role in the packing of crystals in the solid state by means of intermolecular interaction and it has significant influence on dipole moment, polarizability, electronic structure and vibrational modes. ${ }^{17}$ The Mulliken charge analyses of molecules 4CAM and 4CAN are calculated at the HF and DFT/ 
Table 1. Comparison of optimized molecular geometrical parameters of 4chloroanilinium cation.

\begin{tabular}{|c|c|c|c|c|c|}
\hline \multirow{2}{*}{$\begin{array}{l}\text { Atom } \\
\text { Connected }\end{array}$} & \multicolumn{2}{|c|}{ 4CAN } & \multicolumn{2}{|c|}{ 4CAM } & \multirow[b]{2}{*}{ Experimental } \\
\hline & $\mathrm{HF}$ & DFT & $\mathrm{HF}$ & DFT & \\
\hline \multicolumn{6}{|l|}{ Bond length $(\AA)$} \\
\hline $\mathrm{C} 1-\mathrm{C} 2$ & 1.381 & 1.391 & 1.381 & 1.391 & $1.370(2)$ \\
\hline C1-C6 & 1.384 & 1.392 & 1.384 & 1.392 & $1.373(2)$ \\
\hline C1-H7 & 1.074 & 1.082 & 1.074 & 1.082 & 0.930 \\
\hline C2-C3 & 1.381 & 1.391 & 1.381 & 1.391 & $1.368(2)$ \\
\hline C2-CL11 & 1.745 & 1.758 & 1.745 & 1.758 & $1.728(2)$ \\
\hline C3-C4 & 1.383 & 1.392 & 1.384 & 1.392 & $1.373(2)$ \\
\hline C3-H8 & 1.074 & 1.082 & 1.074 & 1.082 & 0.930 \\
\hline C4-C5 & 1.388 & 1.397 & 1.388 & 1.397 & 1.363 (19) \\
\hline C4-H9 & 1.076 & 1.085 & 1.076 & 1.085 & 0.930 \\
\hline C5-C6 & 1.387 & 1.397 & 1.388 & 1.397 & 1.363 (19) \\
\hline C5-N16 & 1.418 & 1.426 & 1.415 & 1.425 & $1.456(3)$ \\
\hline C6-H10 & 1.076 & 1.085 & 1.076 & 1.085 & 0.930 \\
\hline N16-H17 & 1.001 & 1.016 & 0.999 & 1.015 & $0.820(4)$ \\
\hline N16-H19 & 1.000 & 1.015 & 1.000 & 1.016 & $0.940(2)$ \\
\hline \multicolumn{6}{|l|}{ Bond Angle $\left({ }^{\circ}\right)$} \\
\hline C2-C1-C6 & 119.7 & 119.5 & 119.8 & 119.6 & $119.4(15)$ \\
\hline C2-C1-H7 & 120.1 & 120.2 & 120.1 & 120.2 & $120.3(11)$ \\
\hline C6-C1-H7 & 120.2 & 120.3 & 119.6 & 119.7 & $120.3(11)$ \\
\hline C1-C2-C3 & 120.4 & 120.7 & 120.4 & 120.6 & $121.1(2)$ \\
\hline C1-C2-CL11 & 119.8 & 119.6 & 119.8 & 119.7 & $119.5(11)$ \\
\hline C3-C2-CL11 & 119.8 & 119.7 & 119.8 & 119.7 & $119.5(11)$ \\
\hline C2-C3-C4 & 119.8 & 119.7 & 119.7 & 120.3 & $119.4(15)$ \\
\hline C2-C3-H8 & 120.1 & 120.2 & 120.1 & 119.5 & $120.3(11)$ \\
\hline C4-C3-H8 & 120.1 & 120.3 & 120.1 & 120.3 & $120.3(11)$ \\
\hline C3-C4-C5 & 120.4 & 120.4 & 119.2 & 119.3 & $121.5(2)$ \\
\hline C3-C4-H9 & 119.6 & 119.6 & 119.4 & 125.5 & $120.4(11)$ \\
\hline C5-C4-H9 & 120.0 & 120.0 & 120.1 & 119.4 & $120.4(11)$ \\
\hline C4-C5-C6 & 119.3 & 119.3 & 119.2 & 119.3 & $121.5(2)$ \\
\hline C4-C5-N16 & 120.1 & 120.2 & 120.3 & 120.3 & $19.2(3)$ \\
\hline C6-C5-N16 & 120.5 & 120.4 & 120.5 & 120.4 & $119.2(3)$ \\
\hline C1-C6-C5 & 120.5 & 120.5 & 119.8 & 119.6 & $119.4(15)$ \\
\hline C1-C6-H10 & 119.4 & 119.4 & 120.1 & 120.2 & $120.3(11)$ \\
\hline C5-C6-H10 & 120.1 & 120.1 & 120.1 & 120.3 & $120.3(11)$ \\
\hline C5-N16-H17 & 112.1 & 112.6 & 112.5 & 112.5 & $109.0(3)$ \\
\hline C5-N16-H19 & 112.3 & 112.5 & 112.4 & 112.7 & $112.9(15)$ \\
\hline H18-N16-H19 & 113.3 & 110.4 & 109.2 & 109.1 & $107.0(2)$ \\
\hline \multicolumn{6}{|l|}{ Torsion Angle $\left(^{\circ}\right)$} \\
\hline $\mathrm{C} 6-\mathrm{C} 1-\mathrm{C} 2-\mathrm{C} 3$ & -0.1 & -0.0 & 0 & -0.1 & $1.1(4)$ \\
\hline C6-C1-C2-CL11 & 179.8 & 180.0 & 179.9 & 179.8 & $-178.4(14)$ \\
\hline H7-C1-C2-C3 & 179.9 & 180.0 & 180.0 & 180.0 & 178.9 \\
\hline H7-C1-C2-CL11 & -0.1 & -0.01 & -0.1 & 0 & -1.6 \\
\hline C2-C1-C6-C5 & -0.1 & -0.2 & -0.2 & 0 & $-0.3(4)$ \\
\hline C2-C1-C6-H10 & 179.7 & 179.6 & 179.7 & 180 & $-179.7(5)$ \\
\hline H7-C1-C6-C5 & 179.8 & 179.8 & 179.8 & 179.8 & $179.7(5)$ \\
\hline H7-C1-C6-H10 & -0.3 & -0.4 & -0.4 & -0.2 & $0.3(3)$ \\
\hline C1-C2-C3-C4 & 0.1 & 0.1 & 0.1 & 0 & $-0.5(3)$ \\
\hline C1-C2-C3-H8 & -179.8 & -180.0 & -180 & 180 & -178.9 \\
\hline CL11-C2-C3-C4 & -179.8 & -179.8 & -179.9 & -179.9 & -178.4 \\
\hline CL11-C2-C3-H8 & 0.2 & 0.1 & 0.1 & 0.1 & 1.6 \\
\hline C2-C3-C4-C5 & 0.1 & 0.1 & 0.2 & 0.1 & -0.3 \\
\hline C2-C3-C4-H9 & -179.4 & -180.0 & -179.7 & -179.8 & 179.7 \\
\hline $\mathrm{H} 8-\mathrm{C} 3-\mathrm{C} 4-\mathrm{C} 5$ & -180.0 & -180.0 & -179.8 & -179.8 & 179.7 \\
\hline H8-C3-C4-H9 & 0.5 & 0.4 & 0.3 & 0.4 & 0.4 \\
\hline C3-C4-C5-C6 & -0.4 & -0.3 & -0.4 & -0.4 & -0.5 \\
\hline C3-C4-C5-N16 & -177.6 & -177.1 & -177.5 & -177.1 & -178.7 \\
\hline
\end{tabular}


Table 1. (continued)

\begin{tabular}{lccccc}
\hline \multirow{2}{*}{$\begin{array}{l}\text { Atom } \\
\text { Connected }\end{array}$} & \multicolumn{2}{c}{ 4CAN } & \multicolumn{2}{c}{ 4CAM } & \\
\cline { 2 - 5 } & HF & DFT & HF & DFT & Experimental \\
\hline H9-C4-C5-C6 & 179.2 & 179.5 & 179.5 & 179.6 & 179.5 \\
H9-C4-C5-N16 & 2.0 & 2.7 & 2.4 & 2.8 & 1.3 \\
C4-C5-C6-C1 & 0.4 & 0.3 & 0.4 & 0.3 & $-0.5(3)$ \\
C4-C5-C6-H10 & -179.5 & -179.5 & -179.5 & -179.8 & -179.5 \\
C4-C5-N16-H17 & -32.0 & -29.5 & -29.7 & -31.6 & -29.9 \\
C4-C5-N16-H19 & -154.9 & -153.1 & -153.4 & -155.6 & -151.9 \\
C6-C5-N16-H17 & 150.8 & 153.7 & 153.3 & 151.7 & 151.9 \\
C6-C5-N16-H19 & 28.0 & 30.1 & 29.5 & 27.7 & 29.9 \\
\hline
\end{tabular}

\#Ref ${ }^{16}$

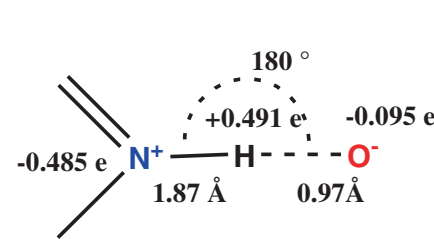

HF

\section{CAN}

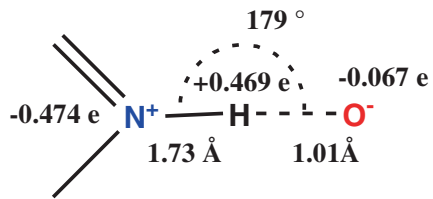

DFT

\section{CAM}

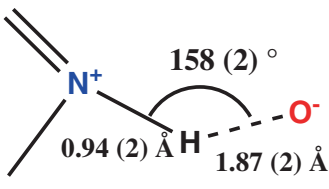

Experimental

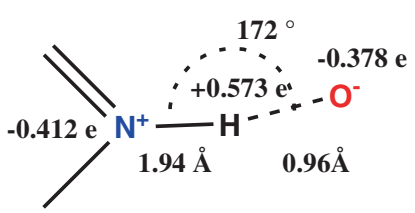

HF

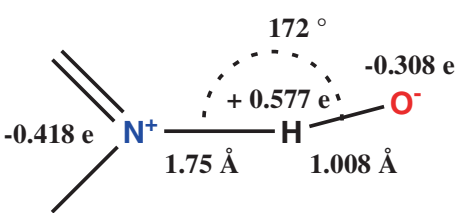

DFT/B3LYP

Scheme 1. Atomic charges and molecular geometry parameters calculated from experimental and theoretical calculations of the $\mathrm{N}-\mathrm{H} \cdots \mathrm{O}$ hydrogen bond.

Table 2. Comparison of optimized molecular geometrical parameters of nitrate anion.

\begin{tabular}{lcc}
\hline & \multicolumn{2}{c}{ Nitrate Anion } \\
\cline { 2 - 3 } Atom Connected & HF & DFT \\
\hline Bond length $(\AA)$ & & \\
N15-O12 & 1.189 & 1.222 \\
N15-O13 & 1.168 & 1.200 \\
N15-O14 & 1.318 & 1.379 \\
O14-H18 & 0.971 & 1.014 \\
Bond Angle $\left(^{\circ}\right)$ & & \\
N15-O14-H18 & 107.6 & 106.1 \\
O12-N15-O13 & 127.6 & 128.0 \\
O12-N15-O14 & 116.9 & 116.9 \\
O13-N15-O14 & 115.5 & 115.1 \\
Torsion Angle $\left({ }^{\circ}\right)$ & & -180.0 \\
O14-O13-N15-O12 & -179.9 & -0.1 \\
H18-O14-N15-O12 & -0.4 & 179.9 \\
H18-O14-N15-O13 & 179.6 & \\
\hline
\end{tabular}

B3LYP levels for the molecule under study which are given in table 4 (a), 4 (b) and 4 (c) and the corresponding population analysis graph is shown in figure 3 . The charge distribution of 4-chloroanilinium cation shows that the five carbon atoms attached to hydrogen atoms (C1 C3, C4, C5 and C6) are negative. Out of five carbon atoms in the 4-chloroanilinium cation, the carbon $\mathrm{C} 5$ atom has more negativity than the others, because the carbon (C5) atom is attached to $\mathrm{NH}_{3}^{+}$group. The elongation of $\mathrm{C}-\mathrm{N}$ bond distance due to the protonation is reiterated with the electronegative repulsion between these two atoms (C5 and N16). Interestingly, another carbon $(\mathrm{C} 2)$ atom from the cation is attached in the chlorine (C111) atom and both of them have electropositive charge. The characteristic feature of $\mathrm{Cl}$ atom is electropositive and it does not participate in any hydrogen bond. In the present case, both the methods predict that the $\mathrm{Cl}$ atom of the cation $(\mathrm{Cl11})$ is positive $(0.277$ $e$ in $\mathrm{HF}$ and $0.340 e$ in DFT) for 4CAM and $(0.275$ $e$ in $\mathrm{HF}$ and $0.335 e$ in DFT) for 4CAN. This may be accorded with the formation of hydrophobic region in the crystal with the presence of the $\mathrm{Cl}$ atoms and the absence of $\mathrm{N}-\mathrm{H} \cdots \mathrm{Cl}$ hydrogen bond.

In the 4CAM anion, all the oxygen atoms have electronegative charges. Out of four oxygen atoms, O19 
Table 3. Comparison of optimized molecular geometrical parameters of maleate anion.

\begin{tabular}{|c|c|c|c|}
\hline \multirow[b]{2}{*}{ Atom Connected } & \multicolumn{2}{|c|}{ Maleate Anion } & \multirow[b]{2}{*}{ Experimental } \\
\hline & $\mathrm{HF}$ & DFT & \\
\hline \multicolumn{4}{|c|}{ Bond Length $(\AA)$} \\
\hline C16-C17 & 1.488 & 1.481 & $1.476(2)$ \\
\hline C16-O19 & 1.199 & 1.230 & $1.267(18)$ \\
\hline $\mathrm{C} 16-\mathrm{O} 20$ & 1.302 & 1.321 & $1.234(19)$ \\
\hline C17-H18 & 1.074 & 1.084 & 0.93 \\
\hline $\mathrm{C} 17-\mathrm{C} 23$ & 1.326 & 1.343 & $1.325(3)$ \\
\hline O19-H21 & 1.716 & 1.635 & 1.207 \\
\hline $\mathrm{H} 21-\mathrm{O} 25$ & 0.954 & 0.990 & 0.930 \\
\hline $\mathrm{C} 22-\mathrm{C} 23$ & 1.514 & 1.509 & $1.476(2)$ \\
\hline $\mathrm{C} 22-\mathrm{O} 25$ & 1.307 & 1.33 & $1.267(18)$ \\
\hline $\mathrm{C} 22-\mathrm{O} 26$ & 1.182 & 1.209 & $1.234(19)$ \\
\hline $\mathrm{C} 23-\mathrm{H} 24$ & 1.075 & 1.085 & 0.93 \\
\hline \multicolumn{4}{|c|}{ Bond Angle $\left(^{\circ}\right)$} \\
\hline C17-C16-O19 & 125.5 & 112.1 & $120.5(14)$ \\
\hline C17-C16-O20 & 111.6 & 122.4 & $117.8(14)$ \\
\hline O19-C16-O20 & 122.9 & 113.4 & $121.7(14)$ \\
\hline C16-C17-H18 & 113.4 & 128.7 & 114.9 \\
\hline C16-C17-C23 & 128.9 & 117.8 & $130.3(8)$ \\
\hline H18-C17-C23 & 117.7 & 110.3 & 114.9 \\
\hline C16-O19-H21 & 111.4 & 109.4 & 115.4 \\
\hline H9-O20-C16 & 110.7 & 169.6 & 111.2 \\
\hline $\mathrm{C} 23-\mathrm{C} 22-\mathrm{O} 25$ & 120.7 & 120.3 & $120.5(14)$ \\
\hline $\mathrm{C} 23-\mathrm{C} 22-\mathrm{O} 26$ & 117.2 & 117.8 & $117.8(14)$ \\
\hline $\mathrm{O} 25-\mathrm{C} 22-\mathrm{O} 26$ & 122.1 & 121.9 & $121.7(14)$ \\
\hline $\mathrm{C} 17-\mathrm{C} 23-\mathrm{C} 22$ & 134.2 & 133.8 & $130.3(8)$ \\
\hline C17-C23-H24 & 116.6 & 116.7 & 114.9 \\
\hline $\mathrm{C} 22-\mathrm{C} 23-\mathrm{H} 24$ & 109.2 & 109.6 & 114.9 \\
\hline $\mathrm{H} 21-\mathrm{O} 25-\mathrm{C} 22$ & 113.4 & 111.8 & 115.4 \\
\hline \multicolumn{4}{|c|}{ Torsion Angle $\left(^{\circ}\right)$} \\
\hline O19-C16-C17-H18 & -179.2 & -179.9 & 179.9 \\
\hline O19-C16-C17-C23 & 0.8 & 0.2 & $0.04(18)$ \\
\hline O20-C16-C17-H18 & 0.8 & 0.1 & 0.2 \\
\hline O20-C16-C17-C23 & -179.2 & -179.9 & $-179.8(9)$ \\
\hline C17-C16-O19-H21 & -0.8 & 0.4 & -0.2 \\
\hline O20-C16-O19-H21 & 179.2 & 179.6 & 179.6 \\
\hline C16-C17-C23-C22 & 0.2 & 0.1 & 0.0 \\
\hline C16-C17-C23-H24 & 180 & 180 & 180 \\
\hline H18-C17-C23-C22 & -179.8 & -179.7 & -180.0 \\
\hline H18-C17-C23-H24 & 0 & -0.4 & 0 \\
\hline $\mathrm{O} 25-\mathrm{C} 22-\mathrm{C} 23-\mathrm{C} 17$ & -0.7 & -0.3 & $0.04(18)$ \\
\hline $\mathrm{O} 25-\mathrm{C} 22-\mathrm{C} 23-\mathrm{H} 24$ & 179.5 & 179.8 & 179.9 \\
\hline O26-C22-C23-C17 & 179.3 & 179.7 & 179.8 \\
\hline O26-C22-C23-H24 & -0.6 & -0.2 & -0.2 \\
\hline C23-C22-O25-H21 & 0.2 & 0.1 & 0.2 \\
\hline O26-C22-O25-H21 & -179.9 & -179.9 & -179.6 \\
\hline
\end{tabular}

$(-0.435 e$ in $\mathrm{HF}$ and $0.332 e$ in DFT $)$ and $\mathrm{O} 25(-0.387$ $e$ in $\mathrm{HF}$ and $-0.274 e$ in DFT) have more electronegativity than the others. These two oxygen atoms behave as a donor and acceptor in the $\mathrm{O}-\mathrm{H} \cdots \mathrm{O}$ intramolecular hydrogen bond and these intramolecular hydrogen bonds are also observed in single-crystal XRD.

The hydrogen atom in the 4CAN anion has more electropositive charges $(0.491 e$ in $\mathrm{HF}$ and $0.469 e$ in
Table 4. (a) Atomic charges for optimized geometry of 4chloroanilinium cation.

\begin{tabular}{|c|c|c|c|c|}
\hline \multicolumn{5}{|c|}{ 4-chloroanilinium cation } \\
\hline \multirow[b]{2}{*}{ Atom } & \multicolumn{2}{|c|}{ 4CAN } & \multicolumn{2}{|c|}{ 4CAM } \\
\hline & $\mathrm{HF}$ & DFT & $\mathrm{HF}$ & DFT \\
\hline $\mathrm{C} 1$ & -0.497 & -0.507 & -0.345 & -0.397 \\
\hline $\mathrm{C} 2$ & 0.468 & 0.606 & 0.482 & 0.623 \\
\hline $\mathrm{C} 3$ & -0.292 & -0.404 & -0.465 & -0.533 \\
\hline $\mathrm{C} 4$ & -0.109 & -0.132 & -0.588 & -0.493 \\
\hline C5 & -0.494 & -0.398 & -0.595 & -0.459 \\
\hline C6 & -0.370 & -0.327 & 0.180 & 0.062 \\
\hline H7 & 0.234 & 0.186 & 0.236 & 0.189 \\
\hline $\mathrm{H} 8$ & 0.237 & 0.186 & 0.233 & 0.185 \\
\hline H9 & 0.205 & 0.159 & 0.187 & 0.148 \\
\hline H10 & 0.190 & 0.151 & 0.202 & 0.151 \\
\hline Cl11 & 0.275 & 0.335 & 0.277 & 0.340 \\
\hline N16 & -0.485 & -0.474 & -0.412 & -0.418 \\
\hline H17 & 0.292 & 0.292 & 0.283 & 0.269 \\
\hline H19 & 0.287 & 0.275 & 0.310 & 0.313 \\
\hline
\end{tabular}

Table 4. (b) Atomic charges for optimized geometry of nitrate anion.

\begin{tabular}{lrr}
\hline Atom & HF & \multicolumn{1}{c}{ DFT } \\
\hline O12 & -0.117 & -0.047 \\
O13 & -0.060 & 0.009 \\
O14 & -0.095 & -0.067 \\
N15 & -0.159 & -0.311 \\
H18 & 0.491 & 0.469 \\
\hline
\end{tabular}

Table 4. (c) Atomic charges for optimized geometry of maleate anion.

\begin{tabular}{lrr}
\hline Atom & \multicolumn{1}{c}{ HF } & \multicolumn{1}{c}{ DFT } \\
\hline H18 & 0.573 & 0.577 \\
C23 & -0.188 & -0.310 \\
C24 & 0.265 & 0.333 \\
H25 & 0.287 & 0.248 \\
O22 & -0.435 & -0.332 \\
O20 & -0.378 & -0.308 \\
H21 & 0.421 & 0.360 \\
C12 & 0.049 & -0.070 \\
C26 & -0.134 & -0.174 \\
H27 & 0.269 & 0.231 \\
O14 & -0.387 & -0.274 \\
O13 & -0.327 & -0.261 \\
\hline
\end{tabular}

DFT) because it is surrounded by two electronegative atoms, viz., nitrogen (N16) and oxygen (O14). This hydrogen atom plays a key role in the solid state through the $\mathrm{N}-\mathrm{H} \cdots \mathrm{O}$ intermolecular hydrogen bond. 

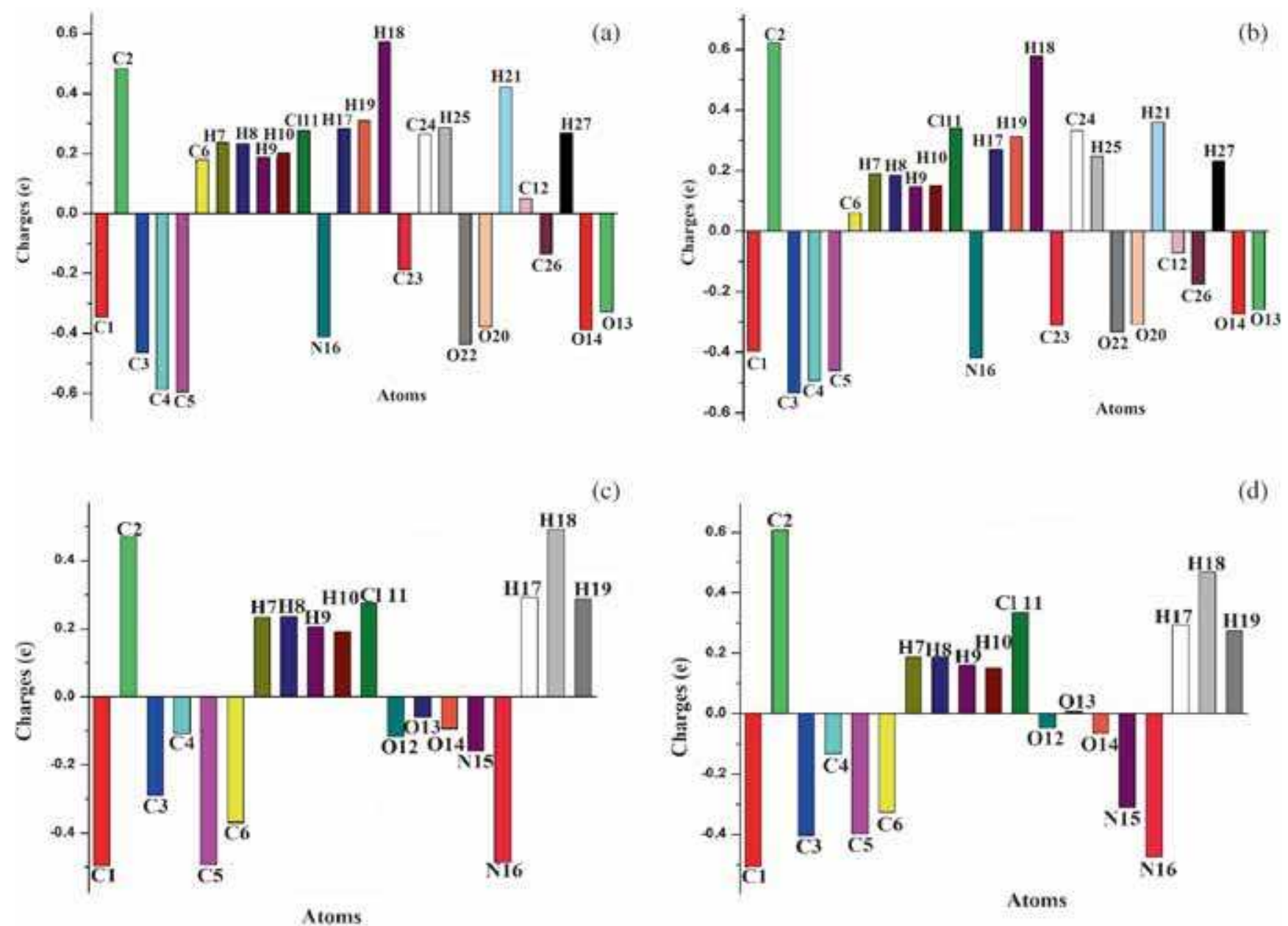

(c)

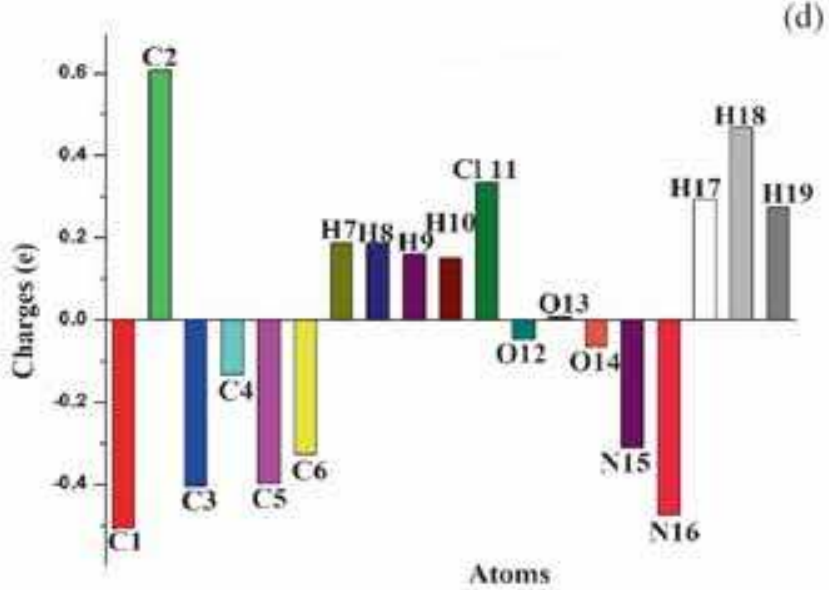

Figure 3. The atomic charges plots by HF (a) and DFT; (b) methods for 4CAM and HF; (c) DFT; (d) methods for 4CAN.

\subsection{Vibrational Analysis}

Vibrational spectroscopy can give valuable information about the hydrogen bonding forces and strength of the intermolecular bonds in addition to symmetry of the individual species. The effect of hydrogen bond is very important in crystal environment. The strong, normal and weak hydrogen bonds cause red shifting of stretching mode vibrations and blue shifting of deformation mode vibrations with different wave numbers depending on their strength. Normally, the vibrational shifts of the stretching modes are greater than that of the deformation modes. This indicates that the linear distortion is much greater than the angular distortion.

The molecular structures of 4CAM and 4CAN have various functional groups such as $-\mathrm{NH}_{3}^{+},-\mathrm{CH},-\mathrm{C}-\mathrm{N}$, $-\mathrm{C}-\mathrm{C}-,-\mathrm{OH},-\mathrm{C}=\mathrm{O}$, disubstituted benzene ring, etc. The vibrational bands of these groups are expected to change in their intensity and position due to their environments. ${ }^{18}$ Complete vibrational analyses of the 72 fundamental modes of 4CAM and 51 fundamental modes of 4CAN are attempted and predicted by the

quantum chemical calculations and compared with their experimental vibrational spectra. The optimized structural parameters are used in the calculation of vibrational frequencies to characterize all stationary points as minimum. The calculated vibrational wave numbers, measured by FT-IR and FT-Raman band positions with their corresponding assignments for the molecules 4CAM and 4CAN, are tabulated in tables 5 and 6, respectively. The FT-IR and FT-Raman spectra of the compounds are shown in figures 4 and 5 , respectively.

3.3a $N-H$ Vibrations: In general, the $\mathrm{N}-\mathrm{H}$ stretching vibrations from the primary amines occur in the region $3600-3300 \mathrm{~cm}^{-1} \cdot{ }^{19,20}$ In the present case, 4choloanilinium cation has more intense peak calculated at $3588 \mathrm{~cm}^{-1}$ and $3504 \mathrm{~cm}^{-1}$ in B3LYP level to assign the $\mathrm{NH}_{2}$ asymmetric and symmetric stretching modes, respectively, for the molecules 4 CAN. Similarly, the asymmetric and symmetric $-\mathrm{NH}_{2}$ stretching occurred as the less-intense peak at 3586 and $3499 \mathrm{~cm}^{-1}$ for the molecule 4CAM. In 4CAN, the medium band observed 
Table 5. Experimental, HF and B3LYP levels computed vibrational frequencies $\left(\mathrm{cm}^{-1}\right)$ obtained for 4-CAN.

\begin{tabular}{|c|c|c|c|c|c|c|c|c|c|}
\hline \multirow[b]{2}{*}{ Mode Number } & \multicolumn{2}{|c|}{ Experimental } & \multicolumn{3}{|c|}{$\mathrm{HF} / 6-311++\mathrm{G}(\mathrm{d}, \mathrm{p})$} & \multicolumn{3}{|c|}{ B3LYP/6-311++G(d,p) } & \multirow[b]{2}{*}{ Assignment } \\
\hline & FT-IR & Raman & $v_{\text {cal }}$ & ${ }^{\mathrm{a}} \mathrm{IR}$ & b $_{\text {I Raman }}$ & $v_{\text {cal }}$ & ${ }^{\mathrm{a}} \mathrm{I}^{\mathrm{IR}}$ & ${ }^{\mathrm{b}_{\mathrm{I}}}$ Raman & \\
\hline 1 & & & 15 & 1.9 & 1.2 & 18 & 1.9 & 2 & Lattice Vibration \\
\hline 2 & & & 30 & 1.3 & 5.2 & 29 & 1.3 & 5.7 & Lattice Vibration \\
\hline 3 & & & 37 & 0.1 & 3.8 & 37 & 0.2 & 4.5 & Lattice Vibration \\
\hline 4 & & & 82 & 0.3 & 0.5 & 79 & 0.2 & 1 & Lattice Vibration \\
\hline 5 & & & 91 & 2 & 1.5 & 99 & 0.7 & 1.2 & Lattice Vibration \\
\hline 6 & & & 120 & 7.7 & 0.4 & 118 & 6.9 & 0.1 & $\omega \mathrm{NH}_{2}+\gamma \mathrm{C}-\mathrm{H}$ \\
\hline 7 & & & 199 & 14.5 & 1.3 & 216 & 37.8 & 2.4 & $\omega \mathrm{NH}_{2}+\gamma \mathrm{C}-\mathrm{H}$ \\
\hline 8 & & & 275 & 0.8 & 0.5 & 258 & 0.8 & 0.4 & $\rho \mathrm{NH}_{2}+\beta \mathrm{C}-\mathrm{H}$ \\
\hline 9 & & & 363 & 2 & 0.6 & 337 & 5 & 0.3 & $\omega \mathrm{NH}_{2}+\gamma \mathrm{C}-\mathrm{H}$ \\
\hline 10 & & & 393 & 9.3 & 0.6 & 377 & 3.5 & 2 & $\rho \mathrm{NH}_{2}+\beta \mathrm{C}-\mathrm{H}$ \\
\hline 11 & & & 411 & 3.1 & 10.2 & 384 & 3.4 & 7.8 & $\beta\left(\mathrm{C}-\mathrm{H}+\mathrm{NH}_{2}\right)$ \\
\hline 12 & & & 454 & 0.1 & 0.2 & 420 & 0.1 & 0.1 & $\gamma \mathrm{C}-\mathrm{H}$ \\
\hline 13 & & & 477 & 4.6 & 0.2 & 465 & 3.2 & 0.2 & $\mathrm{t} \mathrm{NH}_{2}$ \\
\hline 14 & & & 564 & 40.6 & 0.5 & 515 & 33.5 & 0.5 & $\gamma \mathrm{C}-\mathrm{H}$ \\
\hline 15 & & & 690 & 39.3 & 1.8 & 644 & 61.6 & 2.3 & $\beta\left(\mathrm{C}-\mathrm{H}+\mathrm{NH}_{2}\right)$ \\
\hline 16 & & & 696 & 0.3 & 6.1 & 649 & 0.4 & 6.3 & $\beta \mathrm{C}-\mathrm{H}$ \\
\hline 17 & & & 733 & 5.1 & 2.9 & 653 & 0.5 & 4.1 & $\delta \mathrm{O}-\mathrm{N}-\mathrm{O}$ \\
\hline 18 & & & 777 & 7.1 & 0.3 & 688 & 4 & 4.8 & $v \mathrm{~N}=\mathrm{O}+\delta \mathrm{O}-\mathrm{N}-\mathrm{O}$ \\
\hline 19 & $733 \mathrm{w}$ & $734 \mathrm{w}$ & 799 & 15.2 & 2.4 & 718 & 6.9 & 0.7 & $\omega \mathrm{NH}_{2}+\gamma \mathrm{C}-\mathrm{H}$ \\
\hline 20 & & & 859 & 14.9 & 11.4 & 787 & 10.4 & 0.3 & $\gamma \mathrm{O}-\mathrm{N}-\mathrm{O}+\omega \mathrm{NH}_{2}+\gamma \mathrm{C}-\mathrm{H}$ \\
\hline 21 & & & 892 & 4.1 & 18.4 & 813 & 45.1 & 3.8 & $\omega \mathrm{NH}_{2}+\gamma \mathrm{C}-\mathrm{H}$ \\
\hline 22 & $821 \mathrm{~m}$ & $802 \mathrm{~m}$ & 917 & 39.6 & 0.3 & 822 & 2.9 & 1.4 & $\gamma \mathrm{C}-\mathrm{H}$ \\
\hline 23 & & & 922 & 1.2 & 0.5 & 829 & 19.5 & 17.9 & $\gamma \mathrm{C}-\mathrm{H}$ \\
\hline 24 & & & 951 & 81.4 & 0.5 & 904 & 292.5 & 18.1 & $\omega \mathrm{NH}_{2}+\gamma \mathrm{O}-\mathrm{H}$ \\
\hline 25 & & & 1024 & 399.3 & 13.8 & 945 & 160.5 & 11.3 & $\mathrm{t} \mathrm{CH}-\mathrm{CH}+v \mathrm{~N}=\mathrm{O}$ \\
\hline 26 & & & 1064 & 16.3 & 0.6 & 953 & 9.2 & 0.5 & t CH-CH \\
\hline 27 & & & 1088 & 0.3 & 0.1 & 968 & 1.2 & 0.1 & t CH-CH \\
\hline 28 & $1014 \mathrm{w}$ & & 1102 & 36.9 & 0.9 & 1026 & 19.3 & 13.1 & $\beta \mathrm{C}-\mathrm{H}+\gamma \mathrm{O}-\mathrm{H}$ \\
\hline 29 & $1042 \mathrm{w}$ & $1047 \mathrm{~m}$ & 1155 & 95.8 & 14.4 & 1032 & 197.5 & 12.1 & $\omega \mathrm{NH}_{2}+\beta \mathrm{O}-\mathrm{H}$ \\
\hline 30 & $1095 \mathrm{~m}$ & $1094 \mathrm{w}$ & 1160 & 3.7 & 0.9 & 1104 & 50.4 & 29.8 & $\beta \mathrm{C}-\mathrm{H}$ \\
\hline 31 & $1113 \mathrm{w}$ & & 1192 & 5.8 & 0.5 & 1107 & 7.7 & 1.7 & $\rho \mathrm{NH}_{2}+\beta \mathrm{C}-\mathrm{H}$ \\
\hline 32 & $1149 \mathrm{w}$ & $1175 \mathrm{~m}$ & 1197 & 46.1 & 20.7 & 1156 & 6.5 & 1.1 & $\rho \mathrm{NH}_{2}+\beta \mathrm{C}-\mathrm{H}$ \\
\hline 33 & $1202 \mathrm{w}$ & $1209 \mathrm{w}$ & 1289 & 6.7 & 3.2 & 1201 & 6.5 & 5.1 & $\beta \mathrm{C}-\mathrm{H}$ \\
\hline 34 & & & 1317 & 0.4 & 1.2 & 1261 & 118.8 & 22.2 & $v \mathrm{C}-\mathrm{N}+\beta \mathrm{C}-\mathrm{H}$ \\
\hline 35 & & & 1354 & 79.6 & 16.2 & 1319 & 4.1 & 2.2 & $\beta \mathrm{C}-\mathrm{H}$ \\
\hline 36 & & & 1449 & 0.6 & 0.7 & 1328 & 279.5 & 25.4 & $v \mathrm{~N}=\mathrm{O}$ \\
\hline 37 & & & 1567 & 233.1 & 6.2 & 1350 & 1.2 & 0.5 & $\rho \mathrm{NH} 2+\beta \mathrm{C}-\mathrm{H}$ \\
\hline 38 & & & 1573 & 4 & 0.6 & 1458 & 3.3 & 0.1 & $\rho \mathrm{NH} 2+\beta \mathrm{C}-\mathrm{H}$ \\
\hline 39 & $1489 \mathrm{~m}$ & & 1611 & 569.6 & 10.9 & 1497 & 395 & 5 & $\beta \mathrm{O}-\mathrm{H}$ \\
\hline 40 & $1542 \mathrm{~m}$ & $1535 \mathrm{w}$ & 1657 & 126.6 & 1.3 & 1525 & 111.2 & 1 & $\beta \mathrm{C}-\mathrm{H}$ \\
\hline 41 & & $1601 \mathrm{~m}$ & 1772 & 2.1 & 4.6 & 1628 & 2.6 & 3 & $\rho \mathrm{NH}_{2}+v \mathrm{C}-\mathrm{C}+\beta \mathrm{C}-\mathrm{H}$ \\
\hline 42 & & & 1785 & 12.1 & 23.2 & 1641 & 8.7 & 54.7 & $\delta \mathrm{NH}_{2}+v \mathrm{C}-\mathrm{C}+\beta \mathrm{C}-\mathrm{H}$ \\
\hline 43 & & & 1806 & 79.8 & 24.4 & 1661 & 63.1 & 14.6 & $\delta \mathrm{NH}_{2}$ \\
\hline 44 & $1752 \mathrm{~m}$ & & 1928 & 626.4 & 2.9 & 1734 & 280 & 3.5 & $v \mathrm{~N}=\mathrm{O}+\beta \mathrm{O}-\mathrm{H}$ \\
\hline 45 & & $3078 \mathrm{w}$ & 3324 & 11.5 & 71.2 & 2907 & 2497.3 & 364.1 & $v \mathrm{~N}-\mathrm{H} \cdots \mathrm{O}$ \\
\hline 46 & & & 3332 & 5.5 & 60.4 & 3167 & 8.6 & 82 & $v \mathrm{C}-\mathrm{H}$ \\
\hline 47 & & & 3362 & 3.4 & 48.7 & 3172 & 5.5 & 67.6 & $v \mathrm{C}-\mathrm{H}$ \\
\hline 48 & & & 3364 & 0.5 & 142.8 & 3203 & 1.5 & 38.1 & $\nu_{\mathrm{as}} \mathrm{C}-\mathrm{H}$ \\
\hline 49 & & & 3595 & 1529.6 & 158.5 & 3204 & 0.1 & 177.3 & $v_{\mathrm{s}} \mathrm{C}-\mathrm{H}$ \\
\hline 50 & & & 3742 & 36.5 & 113.1 & 3504 & 24.3 & 165.6 & $v_{\mathrm{s}} \mathrm{NH}_{2}$ \\
\hline 51 & & & 3826 & 26.4 & 41.2 & 3588 & 24.9 & 46.4 & $\nu_{\mathrm{as}} \mathrm{NH}_{2}$ \\
\hline
\end{tabular}

w - weak; vs - very strong; $\mathrm{m}$ - medium;

$\nu$, Stretching; $v_{\mathrm{s}}$, sym.stretching; $\nu_{\mathrm{as}}$, asym.stretching; $\beta$ - in-plane bending; $\gamma$ - out-of-plane bending; $\omega$ - Wagging;

$\mathrm{t}$ - twisting; $\delta$ - Scissoring; $\rho$ - Rocking; 
Table 6. Experimental, HF and B3LYP levels computed vibrational frequencies $\left(\mathrm{cm}^{-1}\right)$ obtained for 4-CAM.

\begin{tabular}{|c|c|c|c|c|c|c|c|c|c|}
\hline \multirow[b]{2}{*}{ Mode Number } & \multicolumn{2}{|c|}{ Experimental } & \multicolumn{3}{|c|}{$\mathrm{HF} / 6-311++\mathrm{G}(\mathrm{d}, \mathrm{p})$} & \multicolumn{3}{|c|}{ B3LYP/6-311++G(d,p) } & \multirow[b]{2}{*}{ Assignment } \\
\hline & FT-IR & Raman & $v_{\mathrm{cal}}$ & ${ }^{\mathrm{a}} \mathrm{IR}$ & ${ }^{\mathrm{b}} \mathrm{I}$ Raman & $v_{\text {cal }}$ & ${ }^{\mathrm{a}} \mathrm{I}^{\mathrm{IR}}$ & ${ }^{b} \mathrm{I}$ Raman & \\
\hline 1 & & & 8 & 1.8 & 1.9 & 6 & 1.8 & 2.5 & Lattice Vibration \\
\hline 2 & & & 24 & 3.6 & 2.8 & 26 & 2.7 & 3.8 & Lattice Vibration \\
\hline 3 & & & 27 & 2.3 & 3.3 & 30 & 3.2 & 3.3 & Lattice Vibration \\
\hline 4 & & & 30 & 0.9 & 1.6 & 35 & 0.1 & 3.4 & Lattice Vibration \\
\hline 5 & & & 58 & 4.1 & 1.2 & 64 & 4.4 & 1 & Lattice Vibration \\
\hline 6 & & & 76 & 6.7 & 0.1 & 78 & 7.4 & 0.1 & $\beta(\mathrm{C}-\mathrm{H}+\mathrm{O}-\mathrm{H})$ \\
\hline 7 & & & 87 & 1.2 & 0.6 & 90 & 1.3 & 0.7 & $\beta(\mathrm{C}-\mathrm{H}+\mathrm{O}-\mathrm{H})$ \\
\hline 8 & & & 103 & 4.7 & 2 & 107 & 5.2 & 1.6 & $\gamma \mathrm{C}-\mathrm{H}+\beta(\mathrm{C}-\mathrm{H}+\mathrm{O}-\mathrm{H})$ \\
\hline 9 & & & 181 & 5.1 & 2.2 & 192 & 13 & 4.4 & $\omega \mathrm{NH}_{2}$ \\
\hline 10 & & & 250 & 31 & 0.5 & 256 & 19.6 & 0.6 & $\rho \mathrm{NH}_{2}$ \\
\hline 11 & & & 276 & 0.3 & 0.4 & 260 & 35.6 & 0.5 & $\rho \mathrm{NH}_{2}+\beta \mathrm{O}-\mathrm{H}$ \\
\hline 12 & & & 304 & 11.7 & 1.9 & 297 & 3.8 & 2.8 & $\mathrm{t} \mathrm{CH}-\mathrm{CH}$ \\
\hline 13 & & & 331 & 3 & 5.2 & 299 & 12.3 & 1.9 & $\beta(\mathrm{C}-\mathrm{H}+\mathrm{O}-\mathrm{H})$ \\
\hline 14 & & & 363 & 1.9 & 0.8 & 338 & 4.7 & 0.5 & $\omega \mathrm{NH}_{2}+\gamma \mathrm{C}-\mathrm{H}$ \\
\hline 15 & & & 389 & 10 & 0.5 & 377 & 6 & 2 & $\rho \mathrm{NH}_{2}+\beta \mathrm{C}-\mathrm{H}$ \\
\hline 16 & & & 411 & 3.1 & 9.3 & 384 & 4.4 & 6.3 & $\rho \mathrm{NH}_{2}+\beta \mathrm{C}-\mathrm{H}$ \\
\hline 17 & & & 433 & 4.8 & 4.6 & 409 & 22.9 & 6.5 & $\rho \mathrm{NH}_{2}+\beta \mathrm{O}-\mathrm{H}$ \\
\hline 18 & & & 455 & 0.2 & 0.2 & 420 & 0.5 & 0.1 & $\mathrm{t} \mathrm{CH}-\mathrm{CH}$ \\
\hline 19 & $489 \mathrm{~m}$ & & 475 & 1.2 & 0.5 & 474 & 2 & 0.6 & $\mathrm{t} \mathrm{NH}_{2}$ \\
\hline 20 & & & 567 & 40.9 & 0.6 & 517 & 30.4 & 0.7 & $\omega \mathrm{CH}-\mathrm{CH}$ \\
\hline 21 & & & 649 & 10.2 & 1.7 & 593 & 1.6 & 0.7 & $\omega \mathrm{CH}-\mathrm{CH}$ \\
\hline 22 & $569 \mathrm{~m}$ & & 653 & 2.8 & 0.9 & 601 & 11.2 & 2.9 & $\beta(\mathrm{C}-\mathrm{H}+\mathrm{O}-\mathrm{H})+\omega \mathrm{CH}-\mathrm{CH}$ \\
\hline 23 & & $636 s$ & 671 & 51.1 & 1.6 & 634 & 21.2 & 1.8 & $\beta(\mathrm{C}-\mathrm{H}+\mathrm{O}-\mathrm{H})+\omega \mathrm{CH}-\mathrm{CH}$ \\
\hline 24 & & & 691 & 43 & 2.4 & 646 & 51.4 & 1.7 & $\beta\left(\mathrm{O}-\mathrm{H}+\mathrm{C}-\mathrm{NH}_{2}\right)$ \\
\hline 25 & $694 \mathrm{~s}$ & & 697 & 0.3 & 6.2 & 651 & 0.9 & 5.8 & Ring deformation \\
\hline 26 & & & 782 & 10.8 & 0.8 & 719 & 8.6 & 0.9 & $\omega \mathrm{NH}_{2}+\gamma \mathrm{C}-\mathrm{H}$ \\
\hline 27 & & & 786 & 114.8 & 0.4 & 784 & 17.5 & 1.9 & $\gamma(\mathrm{C}-\mathrm{H}+\mathrm{O}-\mathrm{H})$ \\
\hline 28 & & & 847 & 44.5 & 0.2 & 788 & 21.2 & 0.5 & $\beta(\mathrm{C}-\mathrm{H}+\mathrm{O}-\mathrm{H})$ \\
\hline 29 & $815 \mathrm{w}$ & $806 \mathrm{~s}$ & 867 & 29.8 & 12.7 & 815 & 55.8 & 3.3 & $\omega \mathrm{NH}_{2}+\gamma \mathrm{C}-\mathrm{H}$ \\
\hline 30 & & & 881 & 2.4 & 8.4 & 823 & 4.3 & 2.8 & $\omega \mathrm{CH}-\mathrm{CH}$ \\
\hline 31 & & & 900 & 15 & 16.3 & 830 & 11.5 & 20.3 & $\omega \mathrm{NH}_{2}+\gamma \mathrm{C}-\mathrm{H}$ \\
\hline 32 & & & 913 & 2.3 & 8.5 & 834 & 101.5 & 1.1 & $\gamma \mathrm{O}-\mathrm{H}$ \\
\hline 33 & & & 922 & 0.3 & 0.8 & 850 & 1.4 & 9.4 & $\beta(\mathrm{C}-\mathrm{H}+\mathrm{O}-\mathrm{H})$ \\
\hline 34 & $877 \mathrm{~m}$ & & 964 & 53 & 1.2 & 875 & 25 & 0.8 & $\omega \mathrm{CH}-\mathrm{CH}$ \\
\hline 35 & & 904 w & 973 & 155.2 & 1.6 & 905 & 339 & 25.2 & $\omega \mathrm{NH}_{2}+\gamma \mathrm{C}-\mathrm{H}$ \\
\hline 36 & & & 1025 & 354 & 13.5 & 954 & 13.1 & 1.6 & $\mathrm{t} \mathrm{CH}-\mathrm{CH}$ \\
\hline 37 & & & 1028 & 33.8 & 2.4 & 956 & 36.7 & 3 & $\rho \mathrm{CH}-\mathrm{CH}+\beta \mathrm{O}-\mathrm{H}$ \\
\hline 38 & & & 1065 & 6.6 & 0.3 & 970 & 2.6 & 0.2 & $\mathrm{t} \mathrm{CH}-\mathrm{CH}$ \\
\hline 39 & 999 w & $1013 \mathrm{w}$ & 1089 & 0.3 & 0.1 & 1028 & 23.8 & 2.1 & $\rho \mathrm{CH}-\mathrm{CH}$ \\
\hline 40 & & & 1101 & 31.5 & 1.2 & 1031 & 0.2 & 3.1 & t CH-CH (Maleate) \\
\hline 41 & $1089 \mathrm{w}$ & $1094 \mathrm{~m}$ & 1139 & 0.1 & 7.2 & 1086 & 122.3 & 16.2 & $\rho \mathrm{NH}_{2}+\gamma \mathrm{O}-\mathrm{H}$ \\
\hline 42 & & & 1160 & 4.1 & 0.8 & 1104 & 50.4 & 31.6 & $\beta \mathrm{C}-\mathrm{H}$ \\
\hline 43 & & & 1191 & 6.4 & 0.5 & 1110 & 22.3 & 5 & $\rho \mathrm{NH} 2+\beta \mathrm{C}-\mathrm{H}$ \\
\hline 44 & $1176 \mathrm{w}$ & $1183 \mathrm{w}$ & 1197 & 46.3 & 21.1 & 1156 & 7.8 & 1 & $\rho \mathrm{NH} 2+\beta \mathrm{C}-\mathrm{H}$ \\
\hline 45 & $1213 \mathrm{w}$ & $1208 \mathrm{w}$ & 1289 & 7.6 & 3.3 & 1201 & 6.6 & 5.5 & $\delta \mathrm{CH}-\mathrm{CH}$ \\
\hline 46 & & & 1316 & 3 & 0.7 & 1219 & 90.7 & 12.6 & $\beta \mathrm{C}-\mathrm{H}$ (Maleate) \\
\hline 47 & & $1242 \mathrm{w}$ & 1326 & 85.8 & 13.3 & 1262 & 174.1 & 33.3 & $v \mathrm{C}-\mathrm{N}+\beta \mathrm{C}-\mathrm{H}+\omega \mathrm{NH}_{2}$ \\
\hline 48 & & & 1357 & 109.1 & 17.7 & 1288 & 327.3 & 16.7 & $v \mathrm{C}-\mathrm{O}+\beta(\mathrm{C}-\mathrm{H}+\mathrm{O}-\mathrm{H})($ Maleate $)$ \\
\hline 49 & & & 1395 & 407.1 & 3.4 & 1317 & 40.3 & 21.8 & $\beta(\mathrm{C}-\mathrm{H}+\mathrm{O}-\mathrm{H})($ Maleate $)$ \\
\hline 50 & & & 1432 & 44.9 & 18.7 & 1319 & 5.5 & 1 & $\beta \mathrm{C}-\mathrm{H}$ \\
\hline 51 & $1394 \mathrm{w}$ & $1394 \mathrm{~s}$ & 1449 & 0.1 & 0.8 & 1350 & 1.8 & 0.5 & $\rho \mathrm{NH}_{2}+\beta \mathrm{C}-\mathrm{H}$ \\
\hline 52 & & & 1511 & 12.8 & 5.2 & 1416 & 52.1 & 18.7 & $\beta(\mathrm{C}-\mathrm{H}+\mathrm{O}-\mathrm{H})$ (Maleate) \\
\hline 53 & $1460 \mathrm{~m}$ & & 1558 & 297.3 & 3.7 & 1457 & 94.4 & 0.9 & $\beta(\mathrm{C}-\mathrm{H}+\mathrm{O}-\mathrm{H})$ \\
\hline 54 & & & 1573 & 13.1 & 0.3 & 1459 & 111.9 & 0.5 & $\beta(\mathrm{C}-\mathrm{H}+\mathrm{O}-\mathrm{H})+\rho \mathrm{NH}_{2}$ \\
\hline 55 & & & 1590 & 147.1 & 3.7 & 1492 & 84.8 & 9.3 & $\beta(\mathrm{O}-\mathrm{H}+\mathrm{C}-\mathrm{H})$ (Maleate) \\
\hline 56 & $1579 \mathrm{~m}$ & & 1657 & 153.4 & 1.2 & 1525 & 149.3 & 1 & $\beta \mathrm{C}-\mathrm{H}$ \\
\hline 57 & & $1616 \mathrm{~m}$ & 1771 & 2.9 & 5 & 1628 & 3.3 & 5.7 & $\mathrm{C}-\mathrm{H}$ sextant $+\rho \mathrm{NH}_{2}$ \\
\hline 58 & & & 1785 & 12 & 28.1 & 1641 & 20.2 & 64.3 & $\beta \mathrm{C}-\mathrm{H}+\delta \mathrm{NH}_{2}$ \\
\hline 59 & & & 1807 & 87.7 & 23.2 & 1663 & 74.2 & 13 & $\delta \mathrm{NH}_{2}$ \\
\hline
\end{tabular}


Table 6. (continued)

\begin{tabular}{|c|c|c|c|c|c|c|c|c|c|}
\hline \multirow[b]{2}{*}{ Mode Number } & \multicolumn{2}{|c|}{ Experimental } & \multicolumn{3}{|c|}{$\mathrm{HF} / 6-311++\mathrm{G}(\mathrm{d}, \mathrm{p})$} & \multicolumn{3}{|c|}{ B3LYP/6-311++G(d,p) } & \multirow[b]{2}{*}{ Assignment } \\
\hline & FT-IR & Raman & $v_{\mathrm{cal}}$ & ${ }^{\mathrm{a}} \mathrm{I}^{\mathrm{IR}}$ & ${ }^{\mathrm{b}} \mathrm{I}$ Raman & $v_{\mathrm{cal}}$ & ${ }^{\mathrm{a}} \mathrm{I}^{\mathrm{IR}}$ & ${ }^{\mathrm{b}} \mathrm{I}$ Raman & \\
\hline 60 & & $1685 \mathrm{~m}$ & 1850 & 192.6 & 159.1 & 1680 & 117.6 & 201.6 & $v \mathrm{C}=\mathrm{C}+\beta \mathrm{O}-\mathrm{H}$ \\
\hline 61 & & & 1918 & 405.4 & 39.1 & 1706 & 323.1 & 45 & $v \mathrm{C}=\mathrm{O}+\beta(\mathrm{C}-\mathrm{H}+\mathrm{O}-\mathrm{H})$ \\
\hline 62 & & & 2003 & 497.3 & 95.2 & 1786 & 319.1 & 111.4 & $v \mathrm{C}=\mathrm{O}+\beta(\mathrm{C}-\mathrm{H}+\mathrm{O}-\mathrm{H})$ \\
\hline 63 & $3041 \mathrm{w}$ & $3043 \mathrm{~s}$ & 3324 & 11.7 & 71.1 & 2971 & 3159.4 & 628.1 & $v \mathrm{~N}-\mathrm{H} \cdots \mathrm{O}$ \\
\hline 64 & & $3082 \mathrm{~s}$ & 3332 & 6.3 & 65.3 & 3167 & 8.6 & 82 & $v \mathrm{C}-\mathrm{H}$ \\
\hline 65 & & & 3343 & 0.1 & 42.5 & 3172 & 5.9 & 71.7 & $v_{\mathrm{as}} \mathrm{CH}-\mathrm{CH}$ \\
\hline 66 & & & 3362 & 3.9 & 51.8 & 3173 & 0.3 & 41.9 & $v_{\mathrm{as}} \mathrm{CH}-\mathrm{CH}$ (Maleate) \\
\hline 67 & & & 3362 & 1 & 101.9 & 3190 & 1.5 & 131.6 & $v_{\mathrm{s}} \mathrm{CH}-\mathrm{CH}$ (Maleate) \\
\hline 68 & & & 3363 & 0.4 & 141.7 & 3203 & 1.5 & 63.6 & $v_{\mathrm{s}} \mathrm{C}-\mathrm{H}$ \\
\hline 69 & & & 3725 & 1297.9 & 317.1 & 3204 & 0.2 & 155.1 & $v \mathrm{C}-\mathrm{H}$ \\
\hline 70 & & & 3748 & 358.9 & 88.8 & 3290 & 810.6 & 72.8 & $v$ O-H..O \\
\hline 71 & & & 3831 & 30.4 & 43.4 & 3499 & 30.5 & 172.9 & $v_{\mathrm{s}} \mathrm{NH}_{2}$ \\
\hline 72 & & & 3872 & 582.4 & 35.4 & 3586 & 26.8 & 52 & $\nu_{\mathrm{as}} \mathrm{NH}_{2}$ \\
\hline
\end{tabular}

w - weak; vs - very strong; $\mathrm{m}$ - medium;

$v$, Stretching; $v_{\mathrm{s}}$, sym.stretching; $v_{\mathrm{as}}$, asym.stretching; $\beta$ - in-plane bending; $\gamma$ - out-of- plane bending; $\omega$ - Wagging; $\mathrm{t}-$ twisting;

$\delta$ - Scissoring; $\rho-$ Rocking

at $3078 \mathrm{~cm}^{-1}$ in Raman spectrum is assigned to the N$\mathrm{H}$ stretching. This stretching vibration was calculated

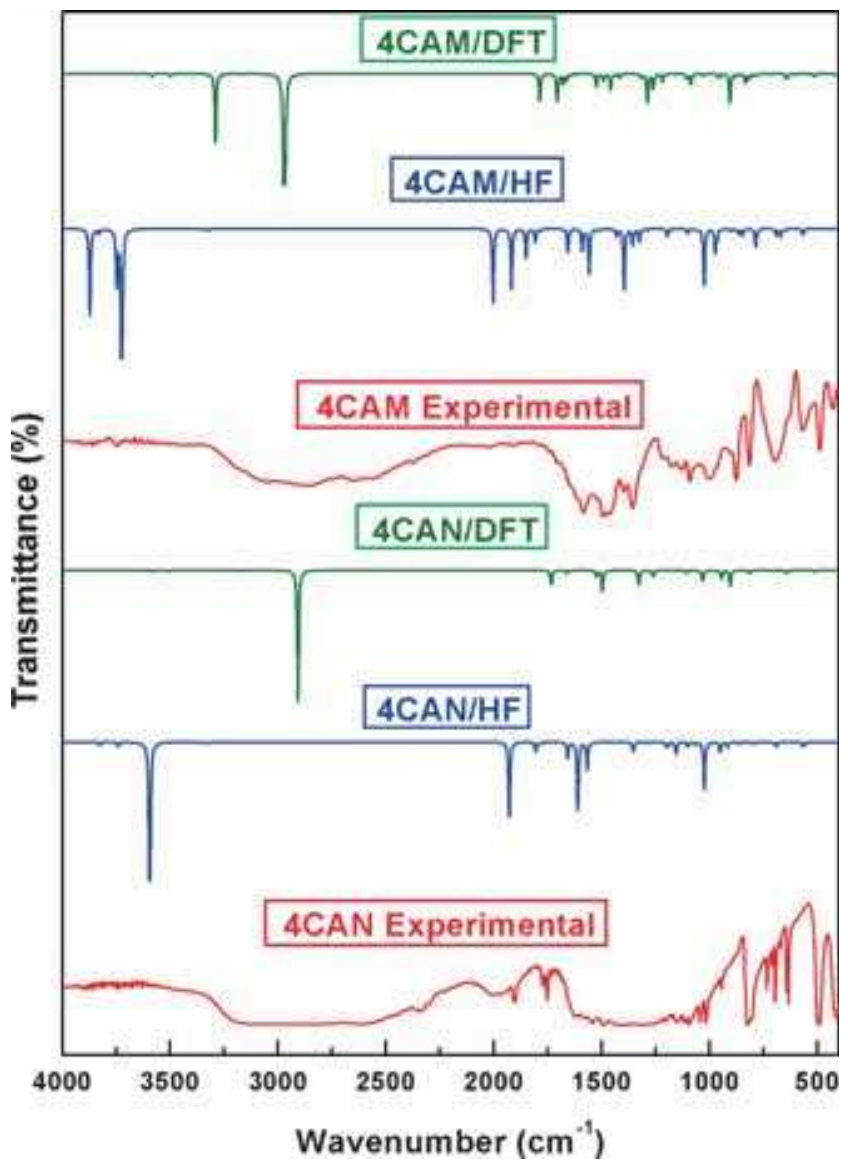

Figure 4. Comparative representation of FT-IR spectra of 4CAM and 4CAN. as a very strong band at 3324 and $2907 \mathrm{~cm}^{-1}$ in $\mathrm{HF}$ and B3LYP level. In 4CAM, the same stretching vibration is

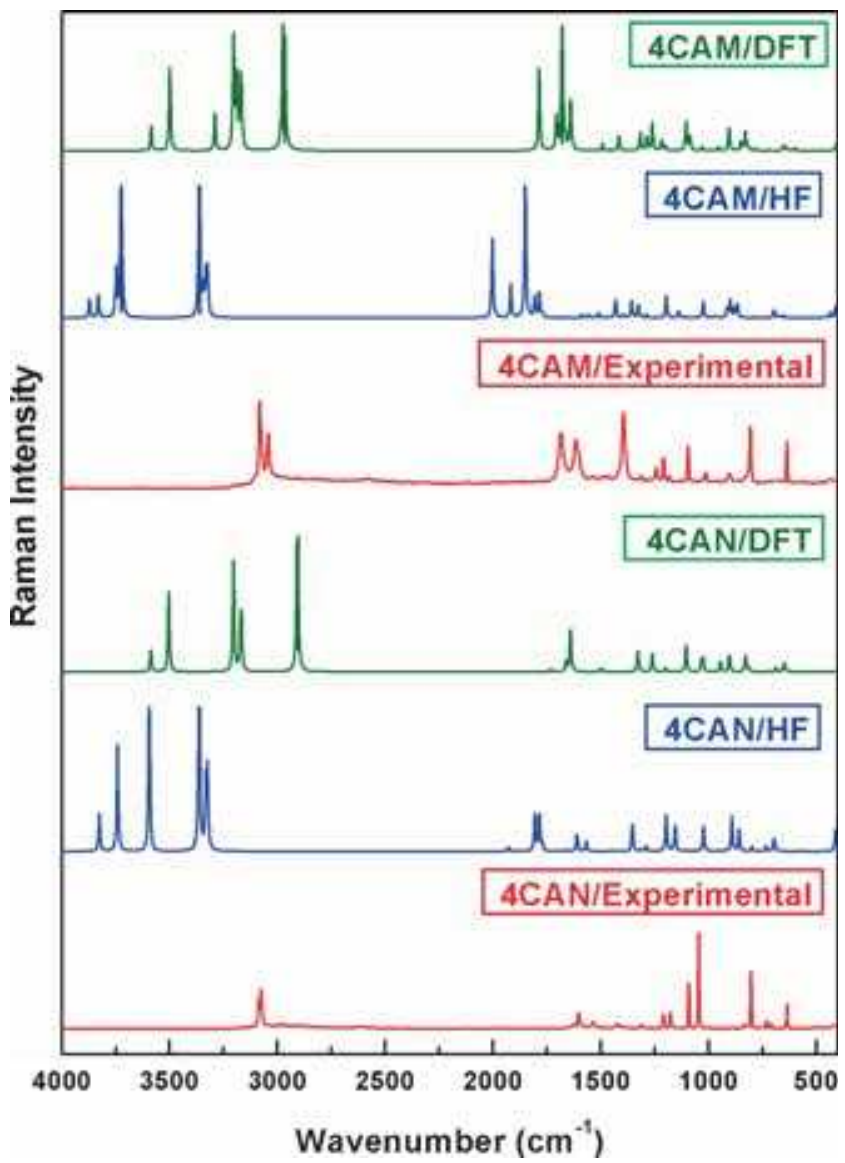

Figure 5. Comparative representation of FT-Raman spectra of $4 \mathrm{CAM}$ and $4 \mathrm{CAN}$. 
observed as a weak band at $3041 \mathrm{~cm}^{-1}$ in IR spectrum and strong band at $3043 \mathrm{~cm}^{-1}$ in Raman spectrum. This corresponding vibration is calculated at 3324 and 2971 $\mathrm{cm}^{-1}$ in HF and B3LYP methods, respectively. These calculated stretching vibrations are occurred as a very strong peak in theoretical methods due to the $\mathrm{N}-\mathrm{H} \cdots \mathrm{O}$ hydrogen bond. But, in the case of experimental IR spectra, the $\mathrm{N}-\mathrm{H} \cdots \mathrm{O}$ hydrogen bond trace is observed as a very broad spectrum around $3200-2200 \mathrm{~cm}^{-1}$ for both the molecules. The occurrences of sharp and broad peaks of the spectrum in the theoretical and experimental methods are due to the fact that the theoretical calculations are carried out in isolated gas state whereas the crystalline state is dominated with the excessive hydrogen bonding interactions. As the hydrogen atom is placed between two heavier electronegative atoms (donor and acceptor) the resulted vibration is observed at a very strong intensity peak for the $\mathrm{N}-\mathrm{H} \cdots \mathrm{O}$ band in theoretical computation. It confirms the relocation of proton from the acids to amino group.

The scissoring vibration of the amine group appears at $1630-1610 \mathrm{~cm}^{-1}$. As one of the $\mathrm{H}$ atoms of the amine group is strongly involved in the $\mathrm{N}-\mathrm{H} \cdots \mathrm{O}$ intermolecular interactions between ion pairs, the $\mathrm{NH}_{3}^{+}$group vibration is reduced to $-\mathrm{NH}_{2}$ molecular vibration. In molecule $4 \mathrm{CAN}, \mathrm{NH}_{2}$ of $\mathrm{NH}_{3}^{+}$scissoring vibration was calculated at 1661 and $1641 \mathrm{~cm}^{-1}$ in DFT/B3LYP method. The vibration coincides with 4 CAM at $1663 \mathrm{~cm}^{-1}$. In both the molecules, the HF level is more deviated from the DFT level. The vibrational mode mentioned above is not observed in the experimental spectra. In 4CAN, the medium band at $1601 \mathrm{~cm}^{-1}$ in Raman spectrum is assigned to the $\mathrm{NH}_{2}$ rocking vibration and the corresponding vibration is predicted at 1772 and $1628 \mathrm{~cm}^{-1}$ in HF and B3LYP method, respectively. In lower wavenumber regions, most of the bands are observed in the scissoring, rocking and wagging vibrations. It is in good agreement with the experimental results.

3.3b $C-H, C-C$ and $C-N$ vibrations: The aromatic $\mathrm{C}-\mathrm{H}$ stretching vibrations are observed in the region $3100-3000 \mathrm{~cm}^{-1}$. $^{21}$ This is the characteristic region for identification of the $\mathrm{C}-\mathrm{H}$ stretching vibrations. Also, the bands are not affected appreciably by the nature of the substituents in this region. The asymmetric $\mathrm{C}-\mathrm{H}$ stretching is calculated at 3595 and $3204 \mathrm{~cm}^{-1}$ in $\mathrm{HF}$ and DFT/B3LYP methods, respectively for 4CAN. In 4CAM, the same vibration is observed at 3725 and 3204 $\mathrm{cm}^{-1}$ in HF and DFT/B3LYP methods respectively. The $\mathrm{C}-\mathrm{H}$ symmetric stretching vibration is predicted at $3364 \mathrm{~cm}^{-1}$ in HF level and $3203 \mathrm{~cm}^{-1}$ in DFT/B3LYP method for $4 \mathrm{CAN}$ and $3363 \mathrm{~cm}^{-1}$ in $\mathrm{HF}$ and $3203 \mathrm{~cm}^{-1}$ in DFT/B3LYP methods for 4CAM. These asymmetric stretching vibrations are not observed in the experimental results. In 4CAM, the strong peak observed at 3082 $\mathrm{cm}^{-1}$ in Raman spectrum is assigned to the $\mathrm{C}-\mathrm{H}$ stretching vibration; this stretching vibration is computed at $3332 \mathrm{~cm}^{-1}$ in the HF level and at $3167 \mathrm{~cm}^{-1}$ in the B3LYP level.

The substituted benzene ring has six $\mathrm{C}-\mathrm{C}$ stretching vibrations. These vibrations mainly involve 'quadrant stretching' of the phenyl $\mathrm{C}-\mathrm{C}$ bonds. But there is a small interaction with $\mathrm{C}-\mathrm{H}$ in-plane bending vibration. There are two quadrant-stretching components in substituted benzenes are expected to appear in the regions $1620-1585$ and $1590-1565 \mathrm{~cm}^{-1}$, respectively. The $1600 \mathrm{~cm}^{-1}$ doublet region is not frequency sensitive to the nature of substitution at ortho, meta and para positions. For these types of substitutions, the quadrant stretching vibrations are infrared inactive because all atoms from the ring are moving to the opposite directions. The $\mathrm{C}-\mathrm{C}$ stretching vibrations mixed with $\mathrm{NH}_{2}$ deformation modes and observed in the modes of 41 and 42 for 4CAN molecule and 57 for 4CAM molecule. In 4CAM, the weak band at $1242 \mathrm{~cm}^{-1}$ in Raman spectrum and IR inactive is assigned to the $\mathrm{C}-\mathrm{N}$ stretching mode. These modes are mixed with $\mathrm{C}-\mathrm{H}$ in-plane deformation modes. The same vibration is computed at 1326 and $1262 \mathrm{~cm}^{-1}$ in the HF and B3LYP methods respectively. It is matched well with the 4CAN molecule.

In general, free $\mathrm{O}-\mathrm{H}$ stretching vibrations appeared around $3600 \mathrm{~cm}^{-1}{ }^{22}$ In the present case, the intense band is computed at $3748 \mathrm{~cm}^{-1}$ and $3290 \mathrm{~cm}^{-1}$ in the HF and DFT/B3LYP methods respectively (table 5). These results show that the DFT/B3LYP method gets downshifted to the literature value which is due to the strong $\mathrm{O}-\mathrm{H} \cdots \mathrm{O}$ intramolecular interaction in the maleate anion. It confirms the migration of the proton transfer between the two $\mathrm{O}$ atoms of the anion. The $\mathrm{O}-$ $\mathrm{H}$...O stretching vibration in the intramolecular hydrogen bonds is crucial in the crystal packing. The medium band observed at $1685 \mathrm{~cm}^{-1}$ in Raman spectrum is assigned to $\mathrm{O}-\mathrm{H}$ in-plane bending vibration. The theoretically computed frequency for $\mathrm{O}-\mathrm{H}$ in-plane bending vibration by DFT/B3LYP method matches well with the experimental results. The medium band at $1460 \mathrm{~cm}^{-1}$ in IR spectrum is assigned to the $\mathrm{O}-\mathrm{H}$ in-plane bending vibration mixed with $\mathrm{C}-\mathrm{H}$ in-plane bending vibration. The $\mathrm{O}-\mathrm{H}$ outof-plane bending vibration is observed at the weak band at $1089 \mathrm{~cm}^{-1}$ in the IR spectrum and the medium band at $1094 \mathrm{~cm}^{-1}$ in the Raman spectrum; this vibration is mixed with $\mathrm{NH}_{2}$ rocking vibration. In lower wavenumber regions most of the bands assigned to the $\mathrm{O}-\mathrm{H}$ in-plane bending vibrations are mixed with the $\mathrm{C}-\mathrm{H}$ in-plane bending vibration. 


\section{3c $O-H$ Vibrations of Maleate Anion:}

3.3d Vibrations of the Nitrate Anion: In the free state the nitrate group has $D_{3 \mathrm{~h}}$ symmetry and its normal modes of vibrations are $A_{1}^{\prime}\left(v_{1}\right), A_{2}^{\prime \prime}\left(v_{2}\right)$ and $E^{\prime}\left(v_{3}\right.$ and $\left.v_{4}\right)$. In which, $v_{1}$ is Raman active, $v_{2}$ is IR active, $v_{3}$ and $v_{4}$ are both IR and Raman active. In the free state of the $\mathrm{NO}_{3}^{-}$anion, the symmetric stretching vibration occurs at $1049 \mathrm{~cm}^{-1}$ the symmetric bending vibration appears at $530 \mathrm{~cm}^{-1}$ and $v_{3}$ and $v_{4}$ (asymmetric stretching and bending modes) occur at 1355 and $690 \mathrm{~cm}^{-1}$, respectively. ${ }^{23-25}$

The medium band at $1752 \mathrm{~cm}^{-1}$ in IR spectrum is assigned to the $\mathrm{N}=\mathrm{O}$ stretching vibration. The same vibration is computed at 1928 and $1449 \mathrm{~cm}^{-1}$ in $\mathrm{HF}$ and 1734 and $1328 \mathrm{~cm}^{-1}$ in DFT/B3LYP methods respectively (table 6). The weak band at $1042 \mathrm{~cm}^{-1}$ in IR spectrum and medium band at $1047 \mathrm{~cm}^{-1}$ in Raman spectrum is assigned to the $\mathrm{N}-\mathrm{O}$ in-plane bending vibration. The corresponding vibration is computed at 1155 and $1032 \mathrm{~cm}^{-1}$ in the HF and DFT/B3LYP methods respectively. The out-of-plane deformation is observed at 859 (HF) and $787 \mathrm{~cm}^{-1}$ (DFT/B3LYP) method. These deformation modes are mixed with the $\mathrm{NH}_{2}$ wagging vibration. These results show migration of proton transfer between the cation and nitrate group of the anion. This out-of-plane deformation is not present in the experimental results.

\subsection{NBO Analysis}

In the NBO analysis, the electronic structures are explained in terms of a set of occupied Lewis and a set of non-Lewis localized orbitals. ${ }^{26}$ Also, delocalization effects can be identified by the presence of off-diagonal elements of the Fock matrix in the NBO basis. The NBO method gives information about interactions in both filled and virtual orbital spaces that could enhance the analysis of intra- and intermolecular interactions.

The delocalization effects of the present molecules can be identified by the diagonal elements of the Fock matrix in the NBO basis. The strengths of these delocalization interactions, $E^{(2)}$, are estimated by second-order perturbation theory. The second-order Fock matrix was calculated to evaluate the donor-acceptor interactions. ${ }^{27}$ These interactions result in loss of occupancy from the localized NBO of the idealized Lewis structure into an empty non-Lewis orbital. For each donor $(i)$ and acceptor $(j)$, the stabilization energy $E^{(2)}$ associated with the delocalization $i \rightarrow j$ is estimated as

$$
E^{(2)}=q_{i} \frac{F(i, j)^{2}}{\left(\varepsilon_{j}-\varepsilon_{i}\right)}
$$

where $q_{i}$ is the donor orbital occupancy, $\varepsilon_{i}$ and $\varepsilon_{j}$ are the orbital energies and $F(i, j)$ is an off- diagonal NBO Fock matrix element. NBO analysis provides an efficient method for studying intra- and intermolecular bonding and interaction among bonds and also provides a convenient basis for investigating charge transfer or conjugative interaction in molecular systems. Some electron donor orbital, acceptor orbital and the interacting stabilization energy resulting from the second-order micro-disturbance theory are reported. ${ }^{28,29}$

NBO analyses of both the molecules have been carried out by the B3LYP/HF method with 6-311++ $\mathrm{G}(\mathrm{d}, \mathrm{p})$ basis set, in order to explain the intramolecular, re-hybridization and delocalization of electron density within the molecules. In the NBO analysis of the hydrogen bonded systems, the most significant element is the charge transfer between the lone pairs of the proton acceptor and the anti-bonds of the proton donor. In case of the present molecular structures, intramolecular charge transfer leads to $\mathrm{C}-\mathrm{C}$ bond in the aromatic ring. As a result of this intramolecular charge transfer, the aromatic ring of the molecules is stabilized, which is due to the $\mathrm{N}-\mathrm{H}$. . O intermolecular hydrogen bonds. The interaction between bonding and anti-bonding of $\mathrm{C}-\mathrm{C}$ bond has large stabilization energy due to the hyperconjugative interactions of $\pi(\mathrm{C}-\mathrm{C}) \rightarrow \pi^{*}(\mathrm{C}-\mathrm{C})$. The intermolecular hyperconjugative interactions $\pi$ $(\mathrm{C} 1-\mathrm{C} 2) \rightarrow \pi^{*}(\mathrm{C} 3-\mathrm{C} 4)$ lead to stabilization of 39.47 $\mathrm{kJ} / \mathrm{mol}$ in $\mathrm{HF}$ and $19.45 \mathrm{~kJ} / \mathrm{mol}$ in DFT method for 4CAN and $38.46 \mathrm{~kJ} / \mathrm{mol}$ in $\mathrm{HF}$ and $18.52 \mathrm{~kJ} / \mathrm{mol}$ in DFT method for 4CAM. This interaction enhances further conjugation with anti-bonding orbital of $\pi *(\mathrm{C} 5-\mathrm{C} 6)$, which leads to strong delocalization energies for both the molecules. The same kind of interaction was identified as $\pi(\mathrm{C} 3-\mathrm{C} 4)$, which is presented in tables TS1 and TS2 (supplementary material) for both the molecules. The lone pair chlorine atom in both molecules has the highest occupancies and the stabilization energies also increase. But in the present case, the chlorine atom does not participate in any intermolecular hydrogen bonds because the chlorine atom has an electropositive charge. Much of the delocalization takes place in the aromatic ring, which is due to the proton transfer between the cation and anion. This larger delocalization of the 4-chloroaninline cation stimulates the bioactivity nature.

\subsection{HOMO and LUMO analysis}

In general, the highest occupied molecular orbital (HOMO) and lowest unoccupied molecular orbital (LUMO) are called as frontier molecular orbitals. These orbitals give information about the chemical hardness, 
electro-negativity and chemical potential of the molecule. ${ }^{30}$ The HOMO and LUMO energies of the molecules 4CAM and 4CAN were calculated by the B3LYP/HF methods with basis set of $6-311++$ $\mathrm{G}(\mathrm{d}, \mathrm{p})$ as listed in table TS3 (supplementary material). The corresponding energy level diagrams are shown in figures 6 and 7, respectively. From these figures, it is observed that the energy levels 320 and 429 in 4CAM and 4CAN are in the energy ranges of -104.843 a.u. to 219.354 a.u. and -101.563 a.u. to 215.760 a.u. in HF and DFT method, respectively, for both molecules. The energy difference between HOMO and LUMO analyses is called as energy gap, which is an important feature for stability of the structures. The calculated energy values of LUMO and HOMO are 0.027 a.u. and -0.328 a.u. for 4 CAM at the HF level and -0.109 a.u. and -0.250 a.u. by the DFT method, -0.028 a.u. and -0.330 a.u. at the HF level, -0.079 a.u. and -0.250 a.u. in DFT level for 4CAN. The frontier molecular orbital energy gap value of 4CAM molecule is 0.355 a.u. at the $\mathrm{HF}$ and 0.141 a.u. at the DFT level and the same energy gap of 4CAN is 0.358 a.u./ 0.171 a.u. in the HF/B3LYP method. The results show that the molecules are having a small frontier energy gap. This small energy gap is associated with a high chemical reactivity, because a huge amount of

(a)
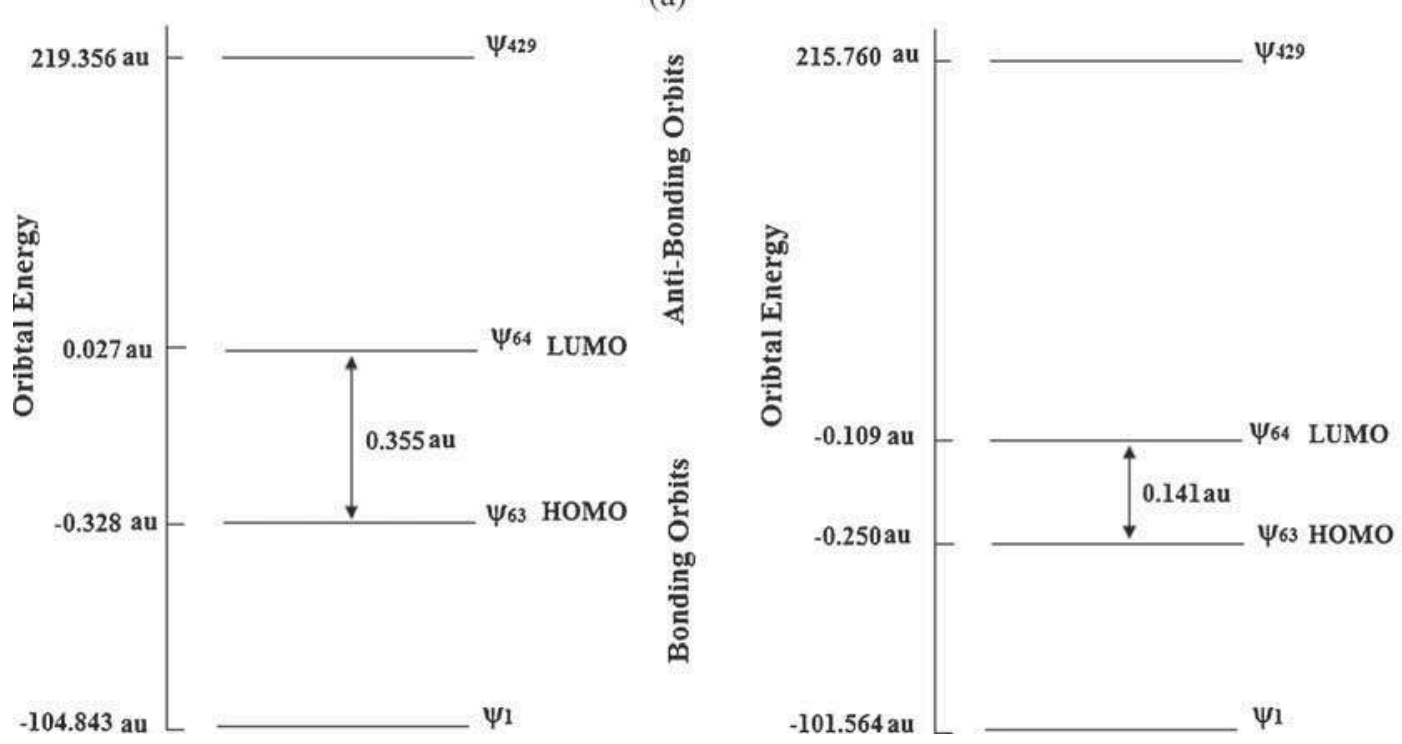

(b)

Figure 6. Energy level diagram of molecular orbits of 4CAM by (a) HF and; (b) B3LYP levels.

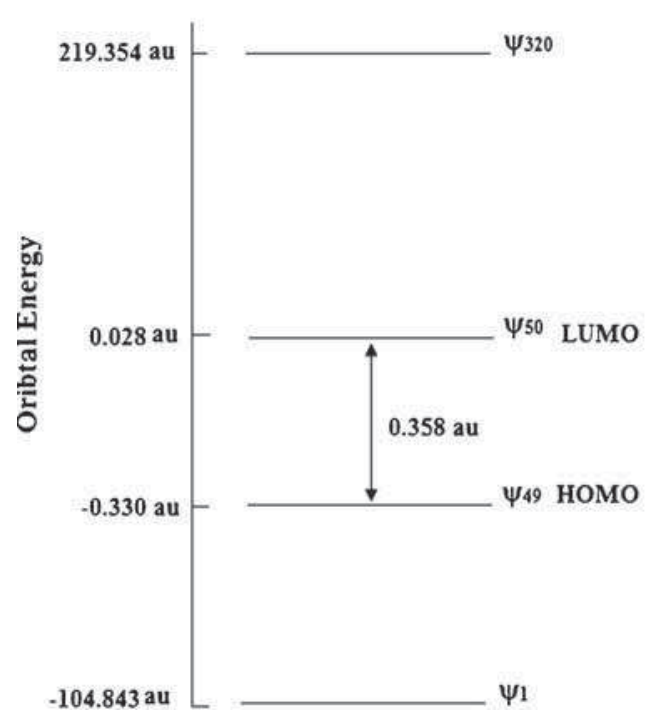

(a)

(b)

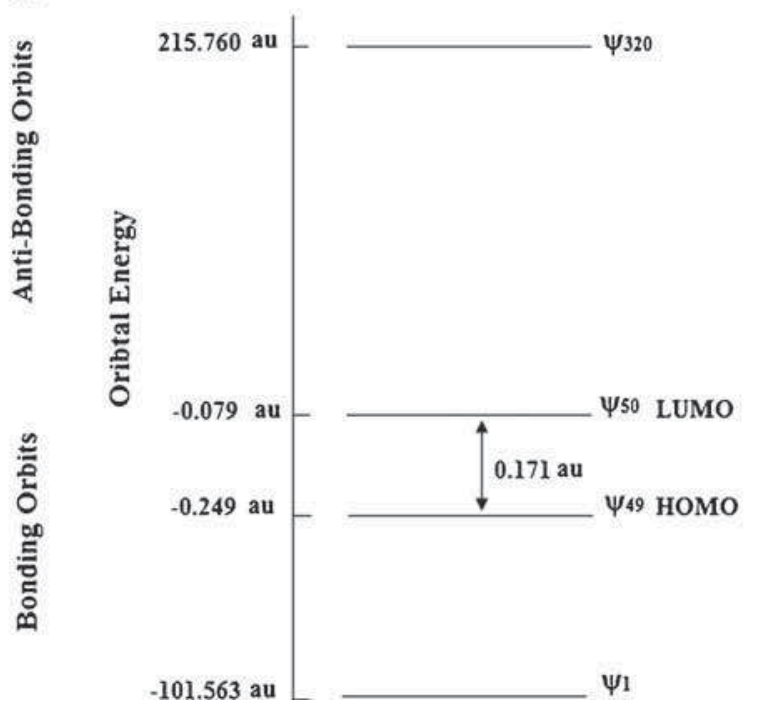

Figure 7. Energy level diagram of molecular orbits of 4CAN by (a) HF and; (b) B3LYP levels. 
charge transfer occurs within the molecule. The narrow energy gap between HOMO and LUMO supports the possible bioactivity of the molecule.

\section{Conclusions}

The experimental and theoretical investigations reveal the proton transfer between the 4-chloroanilinium cation and maleate and nitrate anions. This proton transfer provides the support for the molecular assemblies dominated by the strong inter and intramolecular $\mathrm{N}-\mathrm{H} \cdots \mathrm{O}$ and $\mathrm{O}-\mathrm{H} \cdots \mathrm{O}$ hydrogen bonds. The optimized molecular structure, vibrational frequencies and NBO analysis of 4-chloroanilinium salts were found out by quantum chemical calculations at the HF and DFT/B3LYP methods invoking 6-311++G(d,p) basis sets. The shifting of vibrational bands due to the intermolecular hydrogen bonds was analyzed with experimental and theoretically computed vibrational spectra. Natural bond orbital analysis indicates the strong intermolecular interactions and in an agreement with the experimental intermolecular hydrogen bonding results. Natural bond orbital analysis of the molecule confirms that the charge transfer caused by $\pi$ electron cloud movement from donor to acceptor must be responsible for the bioactivity. The value of the energy gap between the HOMO and LUMO gived the information about chemical softness, chemical hardness, electronegativity and chemical potential of the molecules.

\section{Supplementary Information}

$\mathrm{X}$-ray crystallography data of the compound, 4CAM, is available in Cambridge Structural Database (CSD), Ref No: 877151.

\section{Acknowledgement}

The authors SA and SSK thank the Department of Science and Technology, SERB for the financial support of this work in the form of Fasttrack Research Project scheme.

\section{References}

1. Deechongkit S, Nguyen H, Powers E T, Dawson P E, Gruebele M and Kelly J W 2004 Nature 430 101

2. Lopez N, Vos T E, Arif A M, Shum W W, Noveron J S and Miller J S 2006 Inorg. Chem. 454325

3. Senes A, Ubarretxena-Belandia I and Engelman D M 1998 Proc. Natl. Acad. Sci. USA p. 9056
4. Parker L L, Houk A R and Jensen J H 2006 J. Am. Chem. Soc. 1289863

5. Aakeroy C B and Seddon K R 1993 Chem. Soc. Rev. 22 397

6. Desiraju G R 1989 In Crystal Engineering: The Design of Organic Solids (Amsterdam: Elsevier)

7. Harms K and Wocadlo S 1995 In XCAD4 (Germany: University of Marburg)

8. Sheldrick G M 2008 Acta Cryst. A 64112

9. Farrugia L J 1997 J. Appl. Cryst. 30565

10. Macrae C F, Edgington P R, McCabe P, Pidcock E, Shields G P, Taylor R, Towler M and Van de Streek J 2006 J. Appl. Cryst. 39453

11. Frisch M J, Trucks G W, Schlegel H B, Scuseria G E, Robb M A, Cheeseman J R, Scalmani G, Barone V, Mennucci B, Petersson G A, Nakatsuji H, Caricato M, Li X, Hratchian H P, Izmaylov A F, Bloino J, Zheng G, Sonnenberg J L, Hada M, Ehara M, Toyota K, Fukuda R, Hasegawa J, Ishida M, Nakajima T, Honda Y, Kitao O, Nakai H, Vreven T, Montgomery J A, Peralta J E, Ogliaro F, Bearpark M, Heyd J J, Brothers E, Kudin K N, Staroverov V N, Kobayashi R, Normand J, Raghavachari K, Rendell A, Burant J C, Iyengar S S, Tomasi J, Cossi M, Rega N, Millam J M, Klene M, Knox J E, Cross J B, Bakken V, Adamo C, Jaramillo J, Gomperts R, Stratmann R E, Yazyev O, Austin A J, Cammi R, Pomelli C, Ochterski J W, Martin R L, Morokuma K, Zakrzewski V G, Voth G A, Salvador P, Dannenberg J J, Dapprich S, Daniels A D, Farkas O, Foresman J B, Ortiz J V, Cioslowski J and Fox D J 2013 Gaussian 09, Revision A.1, Gaussian, Inc., Wallingford CT

12. Schlegel H B 1982 J. Comput. Chem. 3214

13. Hohenberg P and Kohn W 1964 Phys. Rev. B. 136864

14. Becke A D 1993 J. Chem. Phys. 985648

15. Dennington R, Keith T and Millam J 2009 J. Semichem Inc., Shawnee Mission KS

16. Anitha R, Athimoolam S, Asath Bahadur $\mathrm{S}$ and Gunasekaran M 2012 Acta Cryst. E 680959

17. Sidir I, Sidir Y G, Kumalar M and Tasal E $2010 \mathrm{~J}$. Mol. Struct. 964134

18. Ploug-Sørensen G and Andersen E K 1983 Acta Cryst. C 39112

19. Kurt M, Yurdakul M and Yurdakul S 2004 J. Mol. Struct. (Theochem.) 71125

20. Krishnakumar V and John Xavier R 2005 Spectrochim. Acta Part A 61253

21. Socrates G 1980 In Infrared Characteristics Group Frequencies (New York: John Wiley)

22. Lampert H, Mikenda W and Karpten A 1997 J. Phys. Chem. A 1012254

23. Banwell C N and Mccash E M 1995 In Fundamentals of Molecular Spectroscopy 4th ed. (New Delhi: Tata McGraw Hill)

24. Dollish F R, Fateley W G and Bentley F F 1973 In Characteristic Raman Frequencies of Organic-Compounds (New York: Wiley)

25. Rosenthal M R 1973 J. Chem. Educ. 50331

26. Reed A E, Curtis L A and Weinhold F A 1988 Chem. Rev. $\mathbf{8 8} 899$ 
27. Szafran M, Komasa A and Adamska E B 2007 J. Mol. Struct. (THEOCHEM) 827101

28. James C, Amal Raj A, Reghunathan Joe I H and Jayakumar V S 2006 J. Raman Spectrosc. 371381
29. Na L J, Rang C Z and Fang Y Z J 2005 Zhejiang Univ. Sci. B 6584

30. Fleming I 1976 In Frontier Orbitals and Organic Chemical Reactions (New York: John Wiley) 